\title{
DNS Investigation of the Dynamical Behaviour of Trailing Vortices in Unbaffled Stirred Vessels at Transitional Reynolds Numbers
}

\author{
S. Başbuğ, G. Papadakis and J. C. Vassilicos \\ Department of Aeronautics, Imperial College London, SW7 $2 A Z$ London, UK
}

\begin{abstract}
Flow in an unbaffled stirred vessel agitated by a four-bladed radial impeller is investigated by using Direct Numerical Simulations (DNS) at $R e=320$ and 1600 . We observe fluctuations in the power consumption with a peak frequency at ca. three times the impeller rotational speed for both Reynolds numbers. It is discovered that these fluctuations are associated with a periodic event in the wake of the blades, which involves alternating growth and decay of the upper and lower cores of the trailing vortex pair as well as up-and-down swinging motion of the radial jet. Moreover, the phase relation between the wakes of the different blades is examined in detail. Further studies using fractal-shaped blades show that the exact blade shape does not have a strong influence on this phenomenon. However, the wake interaction between the blades, hence the number of blades, has a direct influence on the unsteadiness of trailing vortices.
\end{abstract}




\section{INTRODUCTION}

Trailing vortices are observed in the wake of impeller blades used in stirred vessels. Pioneering investigations on this subject date back to early 70 's. Takashima and Mochizuki ${ }^{1}$ placed a camera rotating with the impeller below the tank, in order to visualize the flow field in the rotating reference frame around several impeller types where tracer particles were injected. They provided a schematic diagram illustrating the radial jet on the suction side of a blade as well as the horizontal helical vortices at the upper and lower edges of the blade which give rise to the trailing vortices in the discharge flow. They stated that these trailing vortices were "a kind of Kármán's vortex series". This work was confirmed and improved by the more extensive studies ${ }^{2}$ where a similar measurement technique was applied to examine the flow around a Rushton turbine in a baffled tank. This configuration has been commonly employed for gas-liquid dispersion applications. According to Van't Riet and $\mathrm{Smith}^{3}$, the power consumption and the dispersion performance are mainly determined by the flow in the impeller region. The prominent feature of this flow field is the trailing vortex pair, where gas bubbles are drawn due to low pressure and pass through a region of high shear rate. Their vortex pair idealization slightly differed from that of Takashima and Mochizuki ${ }^{1}$ mainly because of the differences in the impeller geometry, e.g. the inner vertical edge of the Rushton turbine blades. It was observed that the trailing vortices were initially formed at Reynolds number values in the range $150<R e<250$ and it was claimed that the flow around the impeller was turbulent even at $R e=300$, where $R e=N D^{2} / \nu$ based on the rotational speed $(N)$, impeller diameter $(D)$ and kinematic viscosity of the fluid $(\nu)$.

In addition to the determination of the vortex axis location at different Reynolds numbers, Van't Riet and Smith ${ }^{3}$ also derived analytical expressions for the velocity and pressure profiles within the vortex core and found them to be in qualitatively good agreement with their experimental results. On the other hand, the unsteady behaviour of the trailing vortices was only referred to as an "erratic movement". In a later study, Van't Riet et al. $^{4}$ detected that the blade-passage frequency dominates the energy spectra of the velocity fluctuations in the impeller region and these fluctuations were termed as 
pseudo-turbulence. Therefore, it was suggested to obtain the turbulence parameters like the eddy size, turbulence intensity and energy spectra from the measurements acquired in the rotating frame e.g. with probes rotating with the stirrer.

Numerous researchers ${ }^{5-9}$ continued the study of the turbulent flow inside stirred vessels with a focus on trailing vortices. Besides predicting the mean-velocity profiles and turbulence characteristics for the flow around the impeller, they endeavoured to locate the mean path of vortex cores, because many important flow properties are associated with the trailing vortex region. The various predictions of vortex trajectories were compared by Escudié et al. ${ }^{9}$ and collapsed well near the impeller blade, however discrepancies increased with growing distance from the blade. In order to visualise the vortex core, three methods were listed by Escudié and Liné ${ }^{10}$. The first is the determination of the location where the axial velocity is equal to zero. The second utilizes the isosurfaces of vorticity with an arbitrary fixed value to visualise the coherent structures and their centreline can be assumed as the vortex trajectory. A third option is to employ the vortex identification method suggested by Jeong and Hussain ${ }^{11}$, also known as $\lambda_{2}$-criterion. Here $\lambda_{2}$ is the second eigenvalue of the symmetric tensor $\mathbf{S}^{2}+\Omega^{2}$ (assuming $\lambda_{3} \geq \lambda_{2} \geq \lambda_{1}$ ), where $\mathbf{S}$ and $\Omega$ are the symmetric and antisymmetric parts of the velocity gradient tensor, respectively. The region where $\lambda_{2}$ is strictly negative corresponds to the vortex core and an arbitrarily selected threshold can be avoided.

Trailing vortices are the source of turbulence energy inside stirred vessels ${ }^{9}$. Delafosse et al. ${ }^{12}$ and Derksen and Van den Akker ${ }^{13}$ stated that these structures are associated with high levels of turbulence, and emphasized their importance for mixing applications. These aspects motivated extensive studies, mainly concerned with averaged properties such as location and size of vortices, as cited above, or with turbulence properties like anisotropy, time and length scales or dissipation rates ${ }^{14-18}$. On the other hand, the dynamical behaviour of the trailing vortices has received far less attention.

Besides the aforementioned cases with baffled tanks, trailing vortices were also studied in the context of unbaffled tanks ${ }^{19-22}$. Sharp et al. ${ }^{21}$ conducted PIV measurements in an unbaffled tank stirred with a Rushton turbine, and Alcamo et al. ${ }^{22}$ performed 
Large Eddy Simulations (LES) using a similar setup. Both studies (independent from each other) made a comparison between their predictions of trailing vortex trajectories and the published results obtained in baffled tanks. They reported very good agreement between baffled and unbaffled cases. Unbaffled stirred tanks are preferred in food and pharmaceutical industries since vessel cleaning in place is necessary ${ }^{23}$, in bioreactor operations where cell damage is a major concern ${ }^{24,25}$ or in crystallization processes where the baffles can lead to particle attrition ${ }^{26,27}$. When a suspended solid phase is present, a higher mass transfer coefficient between the solid and liquid phases can be obtained in unbaffled vessels with the same power consumption ${ }^{28}$. Moreover, baffles are usually avoided in cases at low Reynolds numbers (in laminar regime) since they can lead to dead zones and deteriorate the mixing performance ${ }^{29}$.

The purpose of the present study is to contribute to the dynamical analysis of the vortex structures behind the impeller blades. To this end, Direct Numerical Simulations (DNS) of the flow field inside an unbaffled stirred vessel with a four-bladed radial impeller have been performed at $R e=320$ and $R e=1600$. The DNS approach was previously utilized to study the flow field in a stirred tank by other authors ${ }^{30,31}$, but their focus was different from that of the present work. Our objective is to characterize the dynamical behaviour, i.e. the periodic features of the trailing vortex structures, which have an impact on the time dependence of the power consumption of a four-bladed radial impeller. Periodic instabilities may also have an influence on the mixing performance ${ }^{32-34}$. However, this study investigates the flow field inside the stirred vessel from a rather fundamental point of view focusing on the instabilities of trailing vortices, and does not target a specific application case.

In this study, two different types of impeller blades are employed, namely regular and fractal-like blades, so that the impact of the blade shape on the trailing vortex dynamics can be assessed. Moreover, in order to understand the role of blade number and wake interaction during the creation of instabilities, cases with one-bladed and two-bladed regular impellers are also investigated. Note that the simulation of the case with eightbladed impeller that was studied by Verzicco et al. ${ }^{30}$, is performed as well, but only for 
code validation purposes.

The remainder of this paper is organized as follows: in Section II we describe the numerical methodology used in this work and present its validation; the flow configuration is introduced in Section III; Section IV is dedicated to the grid convergence study; Sections $\mathrm{V}$ and VI illustrate the examination of the integral balances of angular momentum and kinetic energy, respectively; in Section VII we present the results related to the power draw fluctuations and discuss the link with the trailing vortex structures. Finally Section VIII closes the paper with conclusions.

\section{NUMERICAL METHODOLOGY AND VALIDATION}

The DNS results presented here were obtained with an incompressible in-house code "Pantarhei" which is based on the finite-volume method in unstructured grids, and was previously used for LES of the flow in a stirred vesse ${ }^{35,36}$. The code was parallelized using the PETSc library ${ }^{37}$ and MPI. The PISO algorithm was adopted for the pressure-velocity coupling. Second-order central differencing scheme was selected for the spatial discretization, and implicit second-order Euler method for time-marching. The Navier-Stokes equations were solved in a reference frame rotating with the impeller. Consequently, the momentum equations take the following form:

$$
\frac{\partial \rho \vec{v}}{\partial t}+\nabla \cdot(\rho \vec{v} \otimes \vec{v})=-\nabla p+\nabla \cdot \tau-\rho[\vec{\omega} \times(\vec{\omega} \times \vec{r})+2 \vec{\omega} \times \vec{v}]
$$

where $\vec{v}$ is the velocity vector in the relative frame, $\boldsymbol{\tau}$ is the viscous stress tensor and $\vec{\omega}$ denotes the angular velocity of the frame, in the present paper a vector in the axial direction with the magnitude $\Omega=2 \pi N . \vec{r}$ denotes the position vector with respect to the origin of the coordinate system, which is in the axis of rotation, at the mid-height of the vessel. The last two terms of this equation represent the centrifugal and Coriolis accelerations, respectively, and were treated as source terms during the solution procedure. Since the surrounding walls of the tank have a relative motion with respect to the impeller, a moving wall boundary condition was imposed on the tank walls (i.e. top, bottom and 
side walls). All solid surfaces have a no-slip condition. There is an exception for the top wall for the validation case only (see next paragraph). In all cases considered, the tank is closed at the top, hence there is no free surface and we assume that a wall is present. Also the vessels contain a single phase fluid. When the simulations started from scratch, the fluid was assumed to be at rest in the absolute reference frame, which corresponds to a solid-body rotation in the rotating reference frame. Hence the velocity field over the flow domain was initiated with solid-body rotation. The equations were solved in the Cartesian coordinate system, where the $\mathrm{z}$-axis is the axis of rotation, $\mathrm{x}$ - and $\mathrm{y}$-axes are parallel to the impeller blades (considering four-bladed impellers, as shown in Figure 3b). The velocity components were transformed to a cylindrical coordinate system during the post-processing stage when needed.

It was essential to validate that our approach is capable of successfully predicting the flow field inside a stirred vessel. To this end, the case studied by Verzicco et al. ${ }^{30}$ was employed as a reference and recomputed using our in-house code for comparison of the results. These authors performed DNS of the flow field agitated by an eight-bladed radial impeller inside an unbaffled stirred tank. The flow Reynolds number was $R e=1042$, where $R e=N D^{2} / \nu$. The geometrical details of the tank are displayed in Figure 1 . In the top wall for the validation case, we implemented a free-slip boundary condition (instead of no-slip) in order to match the boundary conditions of the reference work ${ }^{30}$. The grid utilized here was generated to be as similar as possible to that described in the aforementioned paper. The reference work used the immersed boundary method for the modelling of the solid surfaces whereas we utilized a body-fitted mesh; this led to differences in the shape of the cells around the impeller. However, the resolution was kept the same with ca. 2 million cells discretising the domain.

The same paper provided time-averaged and root-mean-square velocity profiles along radial lines at different axial positions which were compared with the experimental results of Dong et al. ${ }^{38}$ who acquired data using the same configuration. Figure 2 illustrates the comparison between these two reference works and our DNS results. The experiments were conducted with a free-surface flow that leads to a certain surface deformation due 


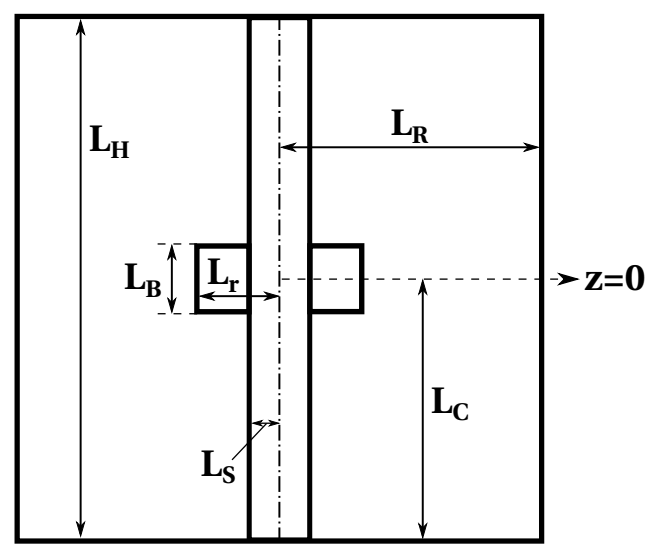

FIG. 1. The configuration investigated by Verzicco et al. ${ }^{30}$ All dimensions are normalized with the impeller radius $L_{r}: L_{S}=0.32, L_{B}=0.8, L_{C}=L_{R}=L_{H} / 2=4$.

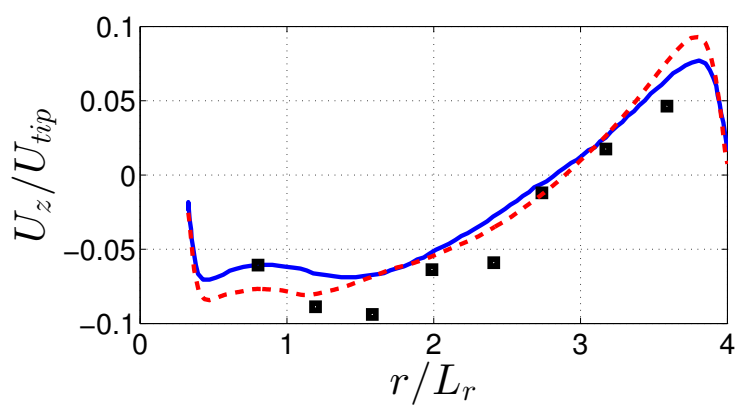

(a) Mean axial velocity.

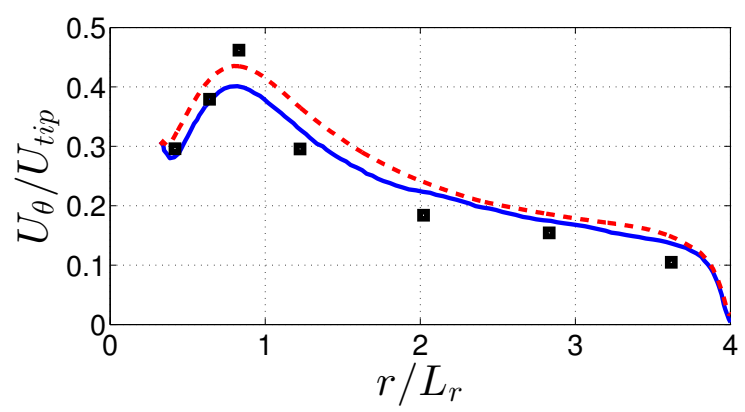

(c) Mean tangential velocity.

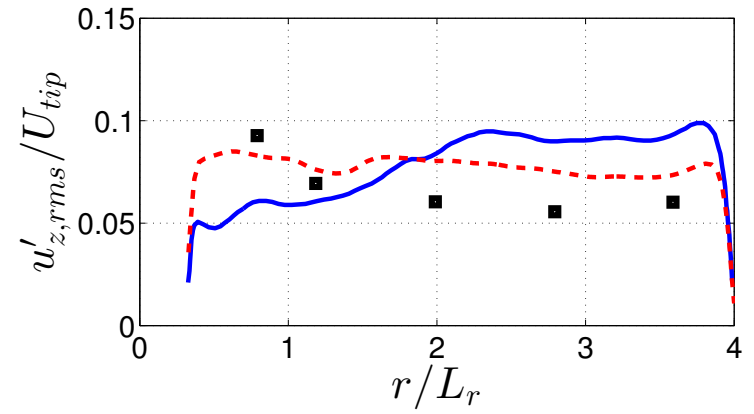

(b) Rms axial velocity.

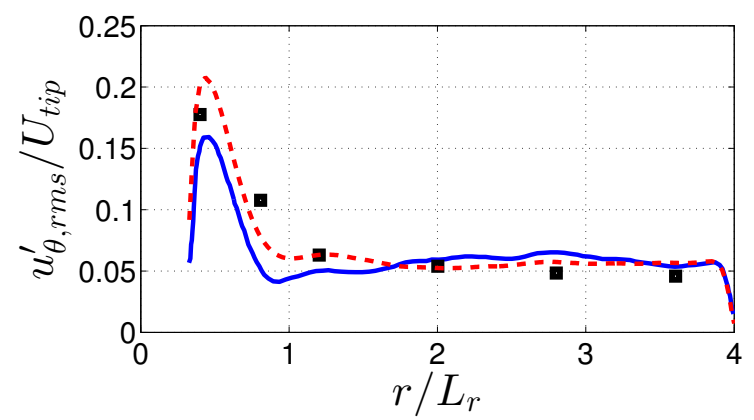

(d) Rms tangential velocity.

FIG. 2. Profiles of radial and tangential velocity components along a radial line in a vertical plane in the middle of two blades, at $z / L_{H}=0.2$. The dashed red lines denote the present DNS results, straight blue lines the DNS of Verzicco et al. ${ }^{30}$ and the square symbols the experimental data of Dong et al. ${ }^{38}$ The velocities are normalized with the blade tip speed $U_{t i p}=\Omega L_{r}$ and radial distance with the impeller radius, $L_{r}$.

to the rotation of the flow. This was neglected by both simulations, which may explain the deviations between the DNS results and the experimental data. Some discrepancies between the two DNS data sets probably stem from the different methodologies used for the modelling of solid surfaces (immersed boundary method vs. body-fitted finite 
volume method). The conventional immersed boundary method as employed by the reference work cannot enforce the no-slip condition exactly on solid surfaces ${ }^{39}$, hence the viscous forces applied by the impeller and tank walls may not be precisely computed leading to some differences in the profiles. As a general conclusion, our DNS results are in satisfactory agreement with the reference numerical predictions and the experimental data. Nevertheless, in the following sections we present a more rigorous analysis of the grid convergence, kinetic energy and momentum balances using the four-bladed impeller.

\section{FLOW CONFIGURATIONS EXAMINED}

The computational domain investigated in the present study has a cylindrical geometry of equal height and diameter, with a four-bladed impeller located at mid-height of the tank. The impeller clearance and diameter with respect to the tank size are shown in Figure $3 \mathrm{a}$ and $3 \mathrm{~b}$. The blade height and blade thickness are 1/10 and 1/100 of the tank height, respectively.

Flows agitated by two types of impeller blades with equal frontal area are examined in this work; the two blade shapes are shown in Figure 3c. The one with the simple rectangular shape is referred to as regular blade, and the other one as fractal blade. In the past decade, the properties of turbulent flows past fractal $/$ multiscale objects have been investigated in detail ${ }^{40-45}$. Many studies have explored their potential benefits in a range of applications, such as the noise reduction ${ }^{46}$, vortex shedding suppression ${ }^{47-49}$, heat transfer enhancement ${ }^{50}$, static mixing improvement ${ }^{51-54}$ and flame control $^{55-57}$ to name a few. Steiros et al. ${ }^{58}$ measured the impeller torque drawn by regular and fractal impellers, identical to the ones used in the present work, and found a torque reduction when fractal blades were employed. Moreover, it was demonstrated by Nedić et al. ${ }^{59}$ in wind tunnel experiments that fractal plates generate higher turbulence intensities in their wake compared to square plates. If accompanied by no increase (or even better by a decrease in power input requirements), this property of fractal plates is potentially very beneficial for mixing applications. Although the analysis of mixing will be the subject of future work, this paper demonstrates the impact of the fractal blades on the flow field 
and the power consumption.

As seen in Figure 3c, the diameter of the fractal impeller is slightly larger or smaller at different heights compared to the regular impeller. Nevertheless, for normalization, the dimensions of the regular impeller are used throughout the paper.

Although the main focus of this work is on four-bladed impellers, we also performed simulations with regular two-bladed and one-bladed impellers to support and complement the results of the four-bladed case. This provided us with the opportunity to assess the effect of the interaction between the blades of the four-bladed impellers as was done in the laboratory experiments of Steiros et al. ${ }^{58}$ In the case of the two-bladed impeller, the angle between the blades is equal to $180^{\circ}$. All the other dimensions remain unchanged.

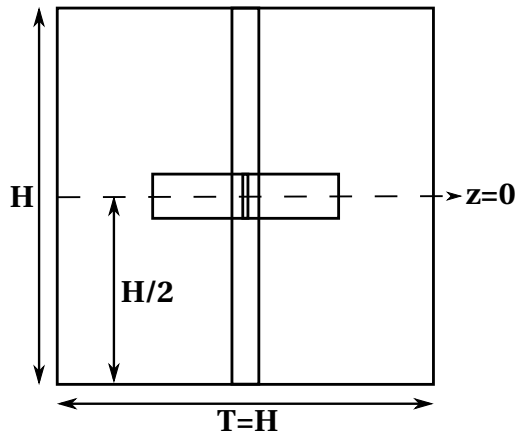

(a)

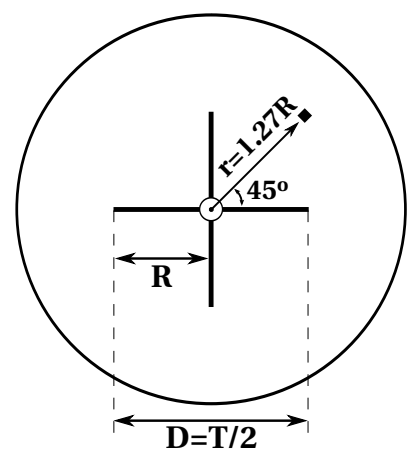

(b)

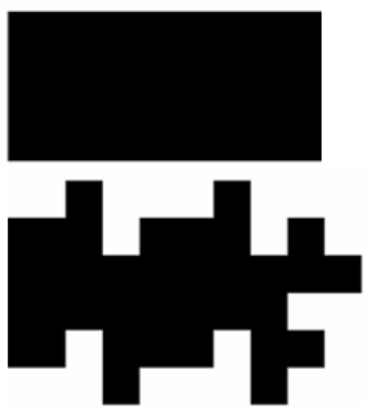

(c)

FIG. 3. Tank geometry, dimensions and blade shapes. a) Vertical view along the axis, b) horizontal view at the mid-height and the location of the probe- 1 marked with the diamond symbol (referred to in Section VII), c) regular (top) and fractal (bottom) blades.

Flows inside stirred vessels with both regular and fractal impellers were simulated at two Reynolds numbers, $R e=320$ and $R e=1600$. Inspection of the power number $\left(\bar{N}_{p}\right)$ variation with $R e$ shows the flow regime in which our simulations are, see Figure 4. The power number stands for the nondimensional power consumption and is defined as $\bar{N}_{p}=\bar{P} /\left(\rho N^{3} D^{5}\right)$, where $\bar{P}$ denotes the time-averaged power draw. The empirical correlation provided by Furukawa et al. ${ }^{60}$ which takes all the geometrical details of the tank into account except the blade thickness, was employed to plot $\bar{N}_{p}$ vs. Re. On the left end of the curve, where Re is very low, there is inverse proportionality between $\bar{N}_{p}$ and $R e$, which is a characteristic of the laminar regime. Fully turbulent regime is observed 
on the right end of the curve and is distinguished by the Re independence of $\bar{N}_{p}$, i.e. a plateau of the curve. Although a fully turbulent flow throughout the tank requires very high Reynolds number ${ }^{61}$, the flow closer to the impeller can become turbulent even at moderate $R e^{31}$. The two Reynolds numbers considered in the present work are indicated in Figure 4 with vertical dashed lines and correspond to the transitional regime. However, some regions of the flow field can still be locally turbulent, especially in the impeller discharge region.

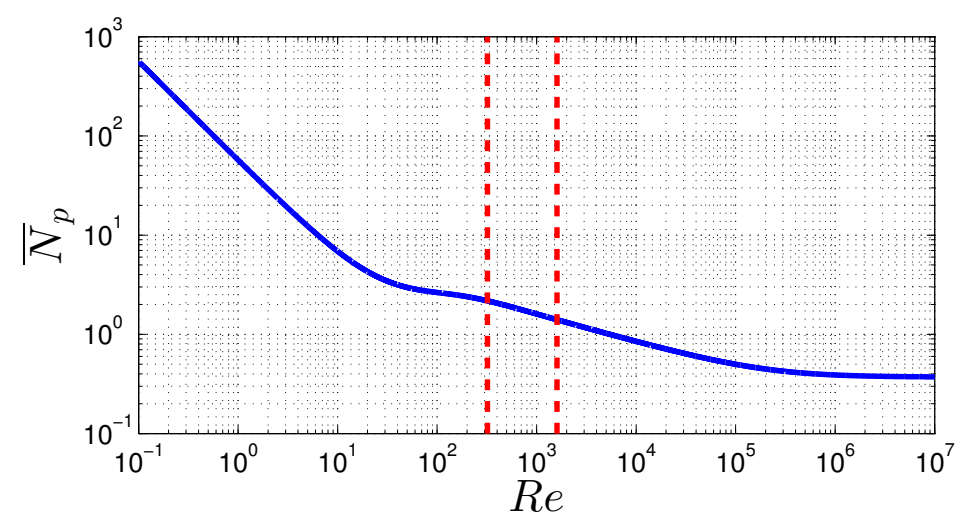

FIG. 4. Power number as a function of Reynolds number based on the empirical correlation given by Furukawa et al. ${ }^{60}$ The vertical dashed lines mark the Reynolds numbers considered in the present work.

\section{GRID CONVERGENCE STUDY}

A grid convergence study was performed for both types of impeller, for $R e=320$. For the regular impeller, the computational domain was discretized using two meshes consisting of $4 \times 10^{6}$ and $13 \times 10^{6}$ hexahedral cells. The simulation was initially run with the coarser grid. Afterwards, the grid was refined, especially near the surfaces and in the impeller discharge region. The developed flow field obtained with the coarse grid was interpolated to the fine grid and served as initial condition. The same procedure was followed for the case of fractal impeller, using meshes with $9 \times 10^{6}$ and $21 \times 10^{6}$ cells. The increased cell numbers for the case of fractal blades is a natural consequence of the more complex blade shape compared to the regular blades. The fine grids used for both cases $\left(13 \times 10^{6}\right.$ or $\left.21 \times 10^{6}\right)$ resolve the blade thickness with 7 cells. The number of cells per 
blade height are 45 for regular and 66 for fractal blades. All four cases were run for a time equivalent to 80 impeller revolutions. Each impeller revolution was resolved using 1500 time steps if one of the coarse grids was used $\left(4 \times 10^{6}\right.$ or $\left.9 \times 10^{6}\right)$ or 3000 time steps if one of the fine grids was used $\left(13 \times 10^{6}\right.$ or $\left.21 \times 10^{6}\right)$. This ensured that the maximum Courant number did not exceed 0.5 in any cell throughout the domain. Figure 5 shows the coarser one of the two grids used for the regular impeller case and the finer one of the two grids used for the fractal impeller case at $R e=320$, in order to exhibit the body-fitted meshing approach employed (only one quarter of the horizontal plane is shown).

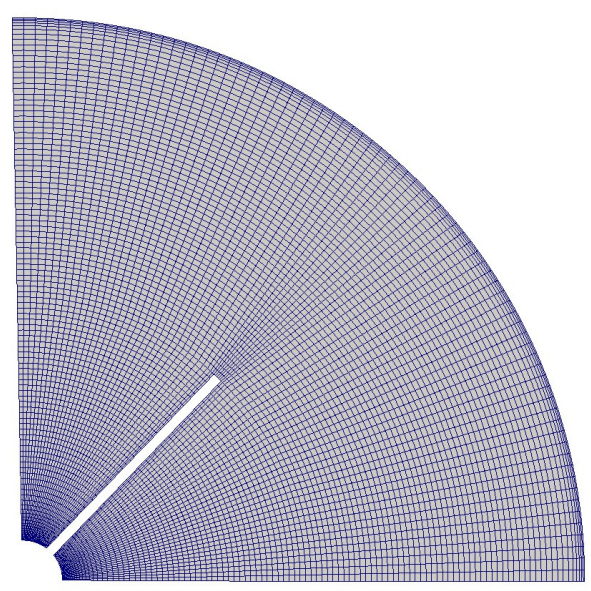

(a) Grid with $4 \times 10^{6}$ cells.

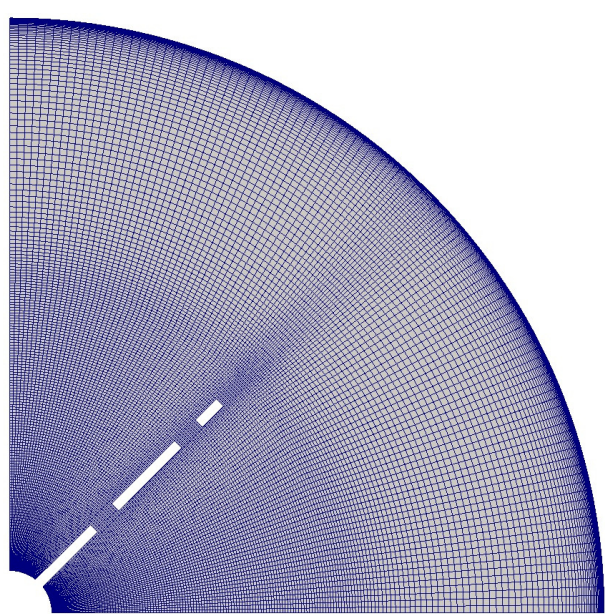

(b) Grid with $21 \times 10^{6}$ cells.

FIG. 5. Examples of the meshing approach for regular (a) and fractal (b) impellers.

Figure 6 exhibits profiles of the mean and rms velocities, turbulence kinetic energy and the dissipation of turbulence kinetic energy, taken from the tank with the fractal impeller. Here, results computed using the grids with $9 \times 10^{6}$ and $21 \times 10^{6}$ cells are compared. The data were extracted along a radial line from the shaft to the tank wall, at the mid-height and at $45^{\circ}$ behind a blade. Since there are four blades, the time-averaged profile was obtained with the average of all four planes (at $45^{\circ}$ behind the blades) to increase the number of samples and improve the statistical convergence. The mean and rms velocities were normalized with the blade tip speed $\left(U_{t i p}=\Omega R\right)$, the turbulence kinetic energy with $U_{t i p}^{2}$, the dissipation of turbulence kinetic energy with $U_{t i p}^{3} / T$ and the radial distance with the blade radius $(R)$. The same factors of normalization were also adopted wherever needed in the remainder of the paper. The tangential velocity component was transformed 
from the relative to the inertial reference frame by adding $\Omega r(x, y)$, where $r(x, y)$ is the distance from the axis of rotation. Since $\Omega r(x, y)$ is constant in time, the rms velocities or turbulence properties did not require a similar transformation.

It can be clearly seen that the profiles of mean velocities collapse very well. The other profiles are also in good agreement, including the profile of the dissipation of turbulence kinetic energy which requires very high resolution to capture correctly. It can be concluded that the refinement from $9 \times 10^{6}$ to $21 \times 10^{6}$ cells did not lead to a significant change in the results and therefore no further refinement was necessary.

Another criterion to assess grid resolution is the ratio of the cell size to the Kolmogorov length scale $(\eta)$. For both grids with $13 \times 10^{6}$ and $21 \times 10^{6}$ cells, this ratio is below 2 throughout the entire tank volume. Also, the wall resolution on the impeller surfaces for these grids is such that the distance between the near-wall cell center and the surface is less than unity in terms of wall units $\left(y^{+}<1\right)$. On the tank walls, the grids are even finer $\left(y^{+}<0.1\right)$. Considering the good agreement of the results obtained with the course and fine grids of the fractal impeller case (shown in Figure 6), we can deduce that the mesh with $13 \times 10^{6}$ cells provides sufficient resolution for the regular impeller case. The results of the cases at $R e=320$ presented for the remainder of this paper belong to the simulations performed using the finer grids, unless otherwise stated.

For $R e=1600$, we used grids with $60 \times 10^{6}$ and $70 \times 10^{6}$ cells for the regular and fractal impellers, respectively. Both grids resolve the blade thickness with 13 cells. The number of cells per blade height are 64 for regular and 96 for fractal blades. One impeller revolution was resolved using 5000 time steps for both cases. To obtain a developed flow field, the simulations at $R e=1600$ were initially run with the grids used at low Re cases for 20 impeller revolutions. Afterwards, the flow field was transferred to the grids designed for the high Reynolds number and the computations were performed for a further 100 impeller revolutions.

We could not afford to make a grid convergence study at this Reynolds number, due to the larger grid sizes and smaller time steps. Nevertheless, the cell size to the Kolmogorov length scale ratio was again kept below 2 for both types of impeller. Moreover, the same 


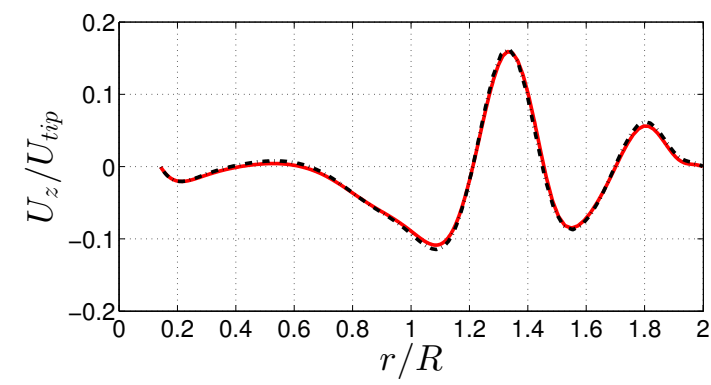

(a) Mean axial velocity.

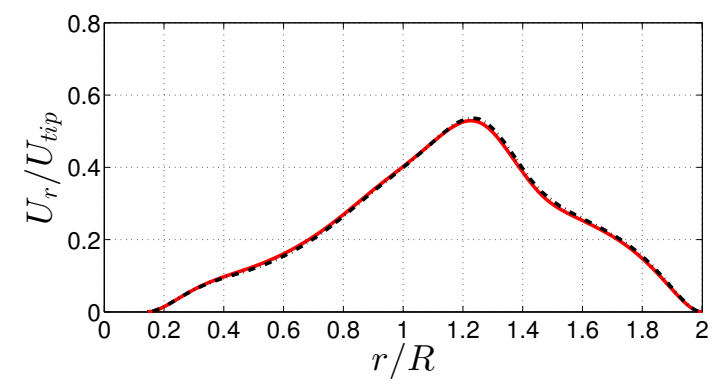

(c) Mean radial velocity.

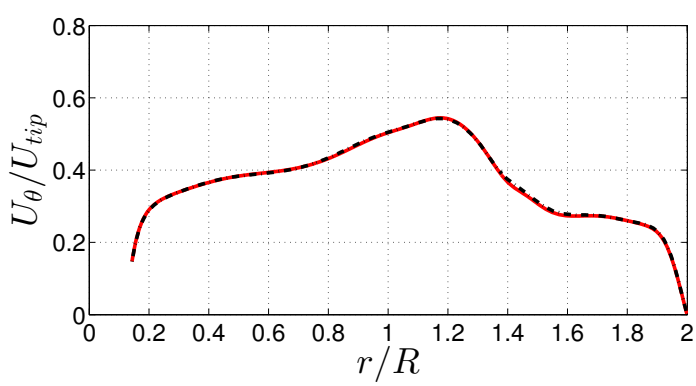

(e) Mean tangential velocity.

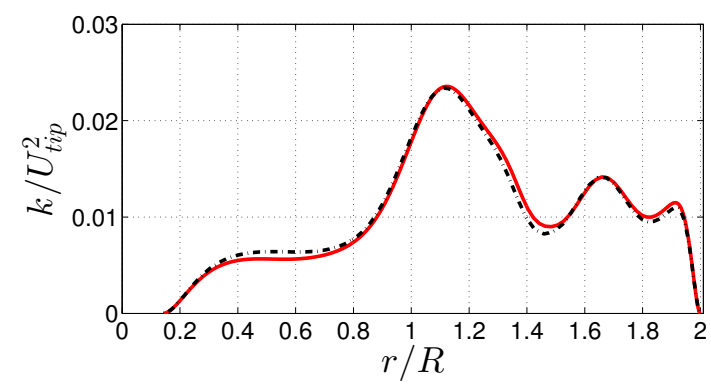

(g) Turbulence kinetic energy.

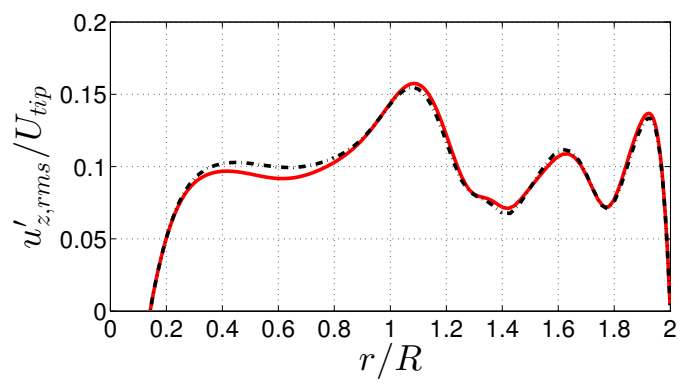

(b) Rms axial velocity.

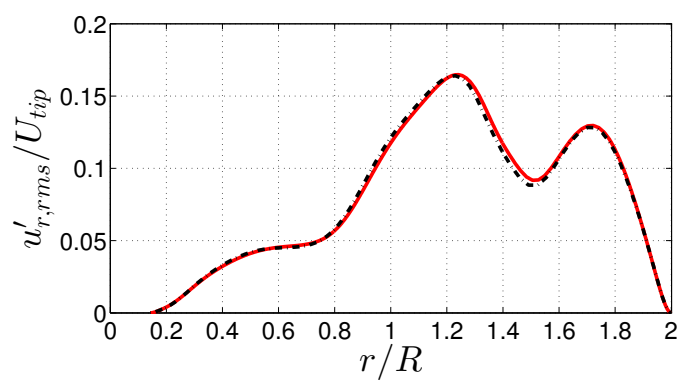

(d) Rms radial velocity.

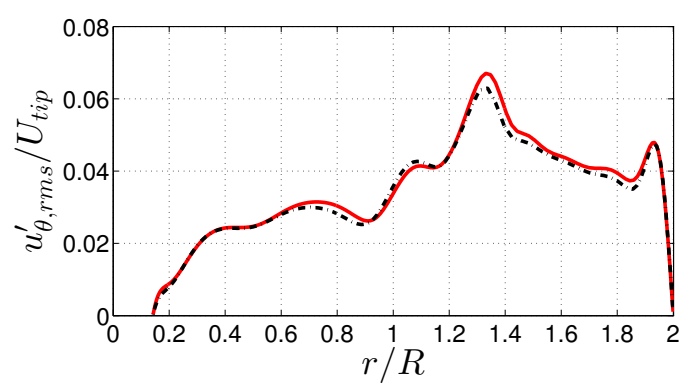

(f) Rms tangential velocity.

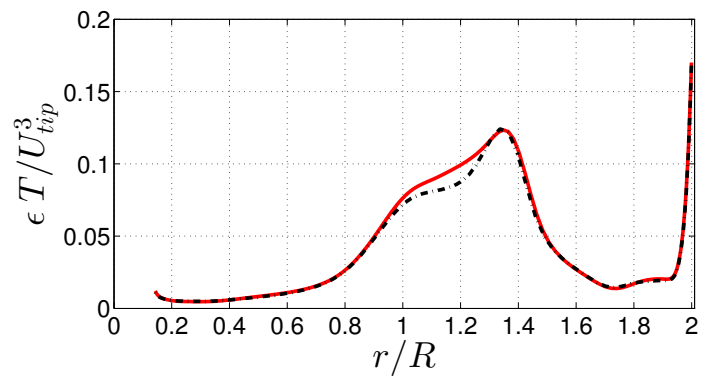

(h) Dissipation of turbulence kinetic energy.

FIG. 6. Profiles along a radial line at the mid-height of the tank with fractal impeller at $45^{\circ}$ behind the blade. Black dashed lines denote results obtained with $9 \times 10^{6}$ cells and red lines with $21 \times 10^{6}$ cells.

wall resolution as the one described above was achieved at the high Reynolds number cases (i.e. $y^{+}<1$ on the impeller surfaces and $y^{+}<0.1$ on the tank walls). 


\section{ANGULAR MOMENTUM BALANCE}

Our incompressible Navier-Stokes finite-volume solver satisfies the conservation of momentum and mass by construction. However, the angular momentum and the kinetic energy balance must be examined separately. The angular momentum of a fluid particle is different for observers in the inertial and rotating reference frames. We evaluate the global angular momentum balance with respect to the center of mass of the entire fluid volume which is located on the axis of rotation. The corresponding conservation equation can be derived by taking the cross product of the location vector $(\vec{r})$ with the NavierStokes equations, already expressed in rotating reference frame (see Equation 1). The integration of the resulting equation over the entire volume takes the following form:

$$
\int_{V} \vec{r} \times\left[\frac{\partial \rho \vec{v}}{\partial t}+\nabla \cdot(\rho \vec{v} \otimes \vec{v})\right] d V=\int_{V} \vec{r} \times[-\nabla p+\nabla \cdot \tau-\rho(\vec{\omega} \times(\vec{\omega} \times \vec{r})+2 \vec{\omega} \times \vec{v})] d V .
$$

We focus on the balance of the axial component of the angular momentum, that is parallel to the axis of impeller rotation, hence directly linked to the power draw. The cross product of $\vec{r}$ with the Coriolis term is projected on the axial direction as follows:

$$
-2[\vec{r} \times(\vec{\omega} \times \vec{v})] \cdot \hat{e}_{z}=-2[\vec{\omega}(\vec{r} \cdot \vec{v})-\vec{v}(\vec{r} \cdot \vec{\omega})] \cdot \hat{e}_{z}=-2 \Omega v_{r}\left(\vec{r} \cdot \hat{e}_{r}\right) .
$$

The final form on the right-hand side of Equation 3 when integrated over the tank volume gives zero. The cross product of $\vec{r}$ with the centrifugal term becomes $(\vec{r} \times \vec{\omega})(\vec{r} \cdot \vec{\omega})$ and has no axial component. The advective term in Equation 2 integrates to zero, as well. The terms related to the pressure gradient and the stress tensor can be reduced to surface integrals. Moreover, the time derivative can be taken out of the integral since the control volume is fixed. The final form (Equation 4) demonstrates that the time derivative of the integrated angular momentum is equal to the net torque applied to the fluid by the solid surfaces (i.e. the impeller, shaft and tank walls).

$$
\frac{\partial}{\partial t} \int_{V}(\vec{r} \times \rho \vec{v}) d V \cdot \hat{e}_{z}=\left[\int_{S}-p(\vec{r} \times \overrightarrow{d S})+\int_{S} \vec{r} \times(\boldsymbol{\tau} \cdot \overrightarrow{d S})\right] \cdot \hat{e}_{z}
$$


Furthermore, we can show that the calculation of the torque applied to the fluid by any surface gives the same result in inertial and rotating reference frames. This is because the pressure field and the viscous stress tensor $(\boldsymbol{\tau})$ are invariant with respect to the transformation between these two frames. To prove this, the relations between the velocities in both frames are written, where $\vec{u}$ and $\vec{v}$ are the velocities in the inertial and the rotating frames, respectively:

$$
u_{r}=v_{r}, u_{\theta}=v_{\theta}+\Omega r, u_{z}=v_{z}
$$

Consequently, the only velocity gradient that can be affected by the transformation is the gradient of the tangential velocity component with respect to the radial direction. There are only two components of the stress tensor associated with this velocity gradient, namely $\tau_{r \theta}$ and $\tau_{\theta r}$. In fact, these are equal due to the symmetry of the tensor:

$$
\tau_{r \theta}=\tau_{\theta r}=\mu\left[r \frac{\partial}{\partial r}\left(\frac{u_{\theta}}{r}\right)+\frac{1}{r} \frac{\partial u_{r}}{\partial \theta}\right]
$$

When $u_{\theta}$ is replaced with $v_{\theta}+\Omega r$ in Equation 6, it can be shown that $\tau_{r \theta}$ computed using the velocities in inertial or relative reference frames are equal, due to the following equality:

$$
\frac{\partial}{\partial r}\left(\frac{u_{\theta}}{r}\right)=\frac{\partial}{\partial r}\left(\frac{v_{\theta}+\Omega r}{r}\right)=\frac{\partial}{\partial r}\left(\frac{v_{\theta}}{r}+\Omega\right)=\frac{\partial}{\partial r}\left(\frac{v_{\theta}}{r}\right)
$$

If averaged for long enough time, the unsteady term in Equation 4 goes to zero since the system is closed and the angular momentum cannot increase indefinitely. Therefore, the net torque applied to the fluid must also be equal to zero in the time-average sense. The blades and the shaft apply torque on the fluid in the positive sense of rotation (for $\Omega>0)$ and the tank walls in the negative sense due to the wall friction. The timederivative term being equal to zero, the torque applied by the impeller and the shaft must balance the oppositely directed torque applied by the tank walls.

This balance was also mentioned by Gimbun et al. ${ }^{62}$ in their paper on the numerical study of turbulent flow in a stirred vessel agitated by a Rushton turbine. Their results indicated an imbalance between the impeller torque and the wall torque of $11 \%$ and $2 \%$ 
obtained by means of Detached Eddy Simulation (DES) and LES methods, respectively. The DES approach is based on LES, but makes use of a one-equation turbulence model near the walls to avoid the high resolution required by LES in this region.

Here, we include the time-derivative term in our assessment of the instantaneous balance (4). The fluid angular momentum is integrated over the tank volume and its time derivative is calculated using a second-order central differencing scheme. The impeller torque is computed from the surface integrals of the pressure and the shear stress applied on the fluid by the blades and the shaft. It is noted that the contribution of the shear stress to the impeller torque for the regular impeller case is only $4 \%$ at $R e=320$ and $0.4 \%$ at $R e=1600$ of the total. The torque applied by the tank walls is likewise calculated with the surface integration of the shear stress. These quantities are normalized with $\left(\rho N^{3} D^{5}\right) / \Omega$, giving rise to the nondimensional torque $\left(N_{T}\right)$.

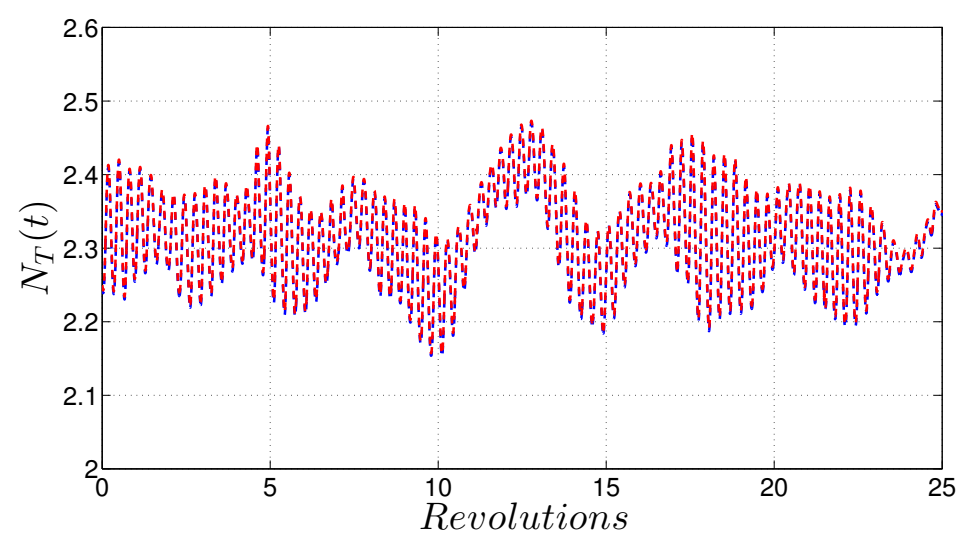

FIG. 7. Torque balance for the regular impeller at $R e=320$. Blue dashed line indicates the impeller torque and red dashed line the sum of the time-derivative term and torque applied by the tank walls.

In Figure 7 the normalized torque is plotted against the number of revolutions, for the case of the regular impeller at $R e=320$. To assess the balance, the torque applied by the impeller is compared with the sum of the other two terms, namely the time-derivative term and the torque applied by the tank walls. The two curves collapse very well indicating that the balance is satisfied instantaneously. The small discrepancy is equal to $0.15 \%$ and is constant in time. All balance results are summarized in Table I.

As stated earlier, the spatial resolution at tank walls is ten times finer in wall units than on the impeller surfaces. This fact results in an accurate computation of the torque 
TABLE I. Torque imbalance for different cases.

\begin{tabular}{l|c|c}
\hline \hline & $R e=320$ & $R e=1600$ \\
\hline Regular impeller & $0.15 \%$ & $0.32 \%$ \\
Fractal impeller & $0.29 \%$ & $1.5 \%$
\end{tabular}

applied by the tank walls. Moreover, the resolution on the tank walls is identical for both regular and fractal impeller cases, whereas the torque imbalance in the fractal impeller case is higher than the regular impeller case, especially at $R e=1600$. This suggests that the small errors most probably stem from the computation of the impeller torque. It is likely that the pressure computed at the edges of the blades include errors due to the high velocity gradients emerging as the fluid particles turn around sharp corners. These gradients increase as the Reynolds number gets higher; hence this conclusion is in accordance with the increased errors at $R e=1600$. It must be noted that the torque applied by the impeller is higher than at the wall, for the fractal impeller at $R e=1600$, and this leads to the difference of $1.5 \%$. Nevertheless, the imbalance is small for all cases. This result gives us confidence about the quality of our predictions of the power drawn by the impeller.

\section{KINETIC ENERGY BALANCE}

In order to examine the kinetic energy balance, the terms of the conservation equation of the total kinetic energy must be evaluated. This equation is derived by taking the inner product of the velocity vector with the incompressible momentum equations. Hence, the aforementioned equation takes the following form (formulated in the inertial frame):

$$
\frac{\partial E}{\partial t}+u_{j} \frac{\partial E}{\partial x_{j}}=-\frac{u_{i}}{\rho} \frac{\partial p}{\partial x_{i}}+\nu u_{i} \frac{\partial^{2} u_{i}}{\partial x_{j}^{2}},
$$

where $E=\frac{1}{2} u_{i} u_{i}$. The last term of the equation, which represents the viscous effects, can be decomposed into diffusive and dissipative parts as:

$$
\nu u_{i} \frac{\partial^{2} u_{i}}{\partial x_{j}^{2}}=\nu \frac{\partial^{2} E}{\partial x_{j}^{2}}-\underbrace{\nu \frac{\partial u_{i}}{\partial x_{j}} \frac{\partial u_{i}}{\partial x_{j}}}_{\tilde{\varepsilon}} .
$$


The last term in Equation 9, i.e. the dissipative term, is called pseudo-dissipation and is symbolized by $\tilde{\varepsilon}$. However, it is common practice to add $\frac{\partial u_{i}}{\partial x_{j}} \cdot \frac{\partial u_{j}}{\partial x_{i}}$ to the Laplace term and subtract the same expression from the pseudo-dissipation term, in order to obtain the following form:

$$
\nu u_{i} \frac{\partial^{2} u_{i}}{\partial x_{j}^{2}}=2 \nu \frac{\partial}{\partial x_{j}}\left(u_{i} S_{i j}\right)-\underbrace{2 \nu\left(S_{i j} S_{i j}\right)}_{\varepsilon} .
$$

$S_{i j}$ is the strain-rate tensor defined as:

$$
S_{i j}=\frac{1}{2}\left(\frac{\partial u_{i}}{\partial x_{j}}+\frac{\partial u_{j}}{\partial x_{i}}\right)
$$

The formulation of the dissipation $(\varepsilon)$ in Equation 10 has the advantage of being the same in both frames considered here, unlike the form referred to as pseudo-dissipation $(\tilde{\varepsilon})$. This is because the strain-rate tensor takes the same values in the two frames considered here, as explained in the previous section. It must be noted that the computed dissipation of total kinetic energy includes not only the turbulence dissipation but also the contribution of the mean velocity gradients. This contribution is insignificant at fully turbulent regime. However, given that we deal with transitional Reynolds numbers, the contribution of the mean velocity gradients may not be negligible in the present case.

The roles of the other terms can be summarized as follows: If the pressure term in Equation 8 is evaluated in the inertial frame and integrated over the entire tank volume, it is the major source of power input. The distribution of the work done by the pressure term is normalized by the time and volume averaged power input $(\bar{P} / V)$. This is shown in Figure 8 displaying that most of the energy is injected in the impeller discharge region. The red regions in this plot illustrate where the flow is accelerated by a favourable pressure gradient, hence the flow kinetic energy is increased. We can see that the rate of energy injection locally is as high as 300 times the volume average of the power input, especially on the suction side of the blades. The blue regions indicate that the flow is also decelerated strongly due to adverse pressure gradient.

The volume integral of the pressure term can be reduced to a surface integral which is non-zero only on the pressure and the suction sides of the impeller blades. The volume 


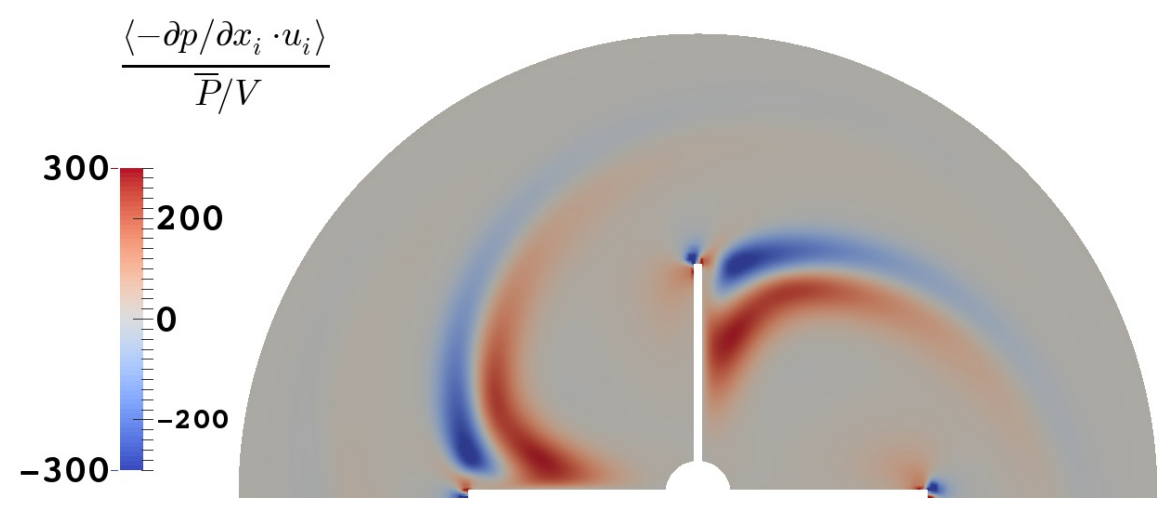

FIG. 8. Contour plot of the work done by the pressure term in the inertial frame at the mid-height of the tank normalized by the time and volume averaged power input, for the regular impeller at $R e=320$. (Since this quantity is computed with time averaging in the relative frame and transformation to the inertial frame, the contour plot is equivalent to a phase-averaged field obtained in the inertial frame.)

integral of the diffusion term can also take the form of a surface integral that quantifies the work done by the viscous forces applied on the surfaces of the impeller and the shaft. The sum of these two terms is equal to the product of the impeller torque with the angular velocity of the impeller $(\Omega)$. The integral of the advective term is equal to zero since the vessel is a closed volume and energy cannot be advected into or out of the tank. The volume integral of the time-derivative term is non-zero at any instant when the power input exceeds the dissipation or vice versa, but its time average converges to zero.

Recall that the Navier-Stokes equations are solved in the rotating reference frame where the extra terms due to Coriolis and centrifugal accelerations are added. However, the Coriolis term is by definition perpendicular to the velocity vector, hence cannot affect the energy balance. The work done by the centrifugal force can locally become the largest term of the equation, but its integral over the volume is equal to zero. Therefore, these two terms do not play a role in the global energy balance. However, there are further differences with respect to the energy balance in the inertial frame. Not only the advection, but also the pressure term integrates to zero, since the blade surfaces are not moving in the rotating frame. The only source of power is the shear stress applied by the moving walls of the tank. As already mentioned, the moving walls apply equal torque to the impeller in time-average sense, and after multiplication with the angular velocity $\Omega$, this 
leads to the same power input as the impeller. In fact, this is in accordance with the earlier determination about the invariance of the dissipation term $\left(2 \nu S_{i j} S_{i j}\right)$. In conclusion, the energy balance is of course also satisfied in the rotating reference frame. The remainder of this section considers the energy balance computed in the inertial frame.

Figure 9 shows the time evolution of the integrated and normalized power input and dissipation over a relatively short time interval. Since any instantaneous difference between the source and sink would lead to an increase or decrease of the total kinetic energy, the time derivative of energy is also integrated over the volume and added to the dissipation, as shown with the green curve. When this is compared with the power injection of the impeller, the two curves (shown with the blue and green lines) agree well, except for a small discrepancy that remains constant in time. The same discrepancy also appears when the time-averaged power input and the time-averaged dissipation are compared. It is possibly due to the numerical dissipation and discussed later in this section. Furthermore, both blue and green curves illustrate synchronized fluctuations with multiple peaks per revolution, similar to that observed in the time evolution of the torque shown in Figure 7. This high frequency fluctuation is less evident in the dissipation but the addition of the time derivative of the energy brings this to the level of the impeller power. Moreover, the plot demonstrates that there is an indication of a superimposed signal with much lower frequency. These aspects are analysed and discussed in the following sections of the present paper.

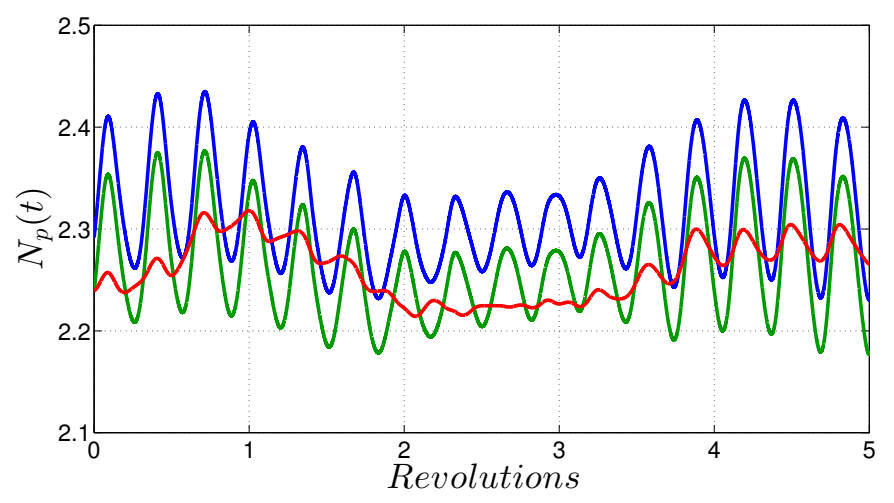

FIG. 9. Power draw $(P(t))$ calculated using impeller torque (blue line), $\int \varepsilon d V$ i.e. integral of dissipation in the whole domain (red line) and $\int(\varepsilon+d E / d t) d V$ (green line), for the regular impeller at $R e=320\left(\varepsilon=2 \nu S_{i j} S_{i j}\right) . N_{p}(t)=P(t) /\left(\rho N^{3} D^{5}\right)$. 
The statistical convergence of the running averaged power number is shown in Figure 10 , for both regular and fractal impellers at $R e=320$. It is observed that any change in the running averaged values of $\bar{N}_{p}$ in the period after 40 revolutions is less than $\pm 0.16 \%$ for the regular and $\pm 0.30 \%$ for the fractal impeller, with respect to the final values of averaging. Hence the averaging is finalized after 80 revolutions and assumed sufficiently converged.

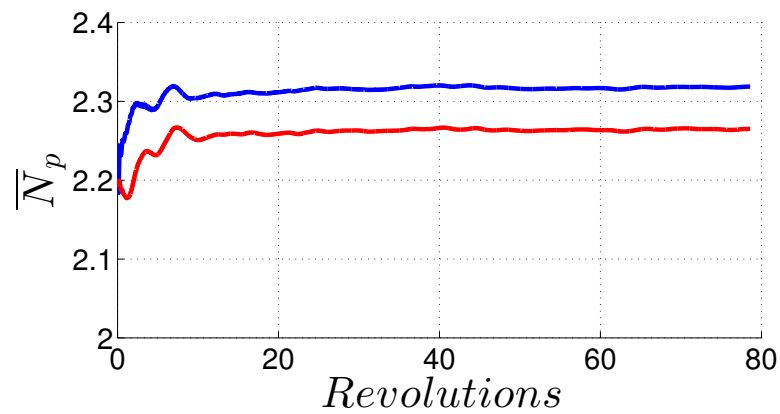

(a) Regular impeller.

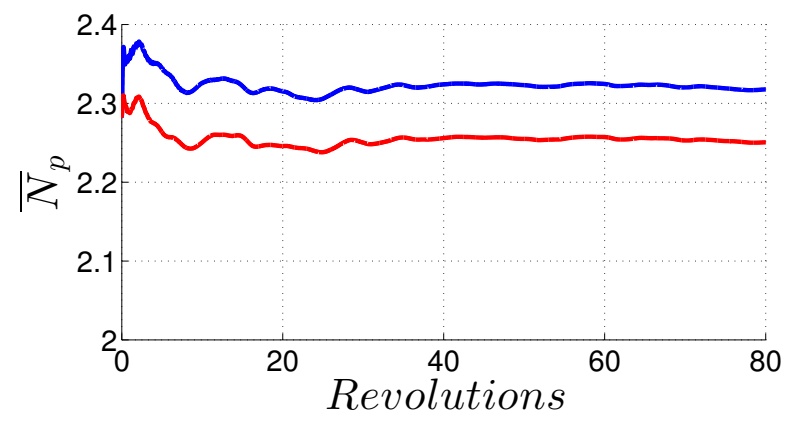

(b) Fractal impeller.

FIG. 10. Running average of the power number over number of revolutions at $R e=320$, based on impeller torque (blue line) and on dissipation (red line).

Table II provides the values of $\bar{N}_{p}$ for the $R e=320$ cases averaged for 80 revolutions and compares the values based on the power input of the impeller and the volume integral of dissipation. The results based on the impeller power and obtained with the fine grids suggest that the power consumption is not affected by the difference in the blade shape for this Reynolds number. Also the relatively coarse grid of $9 \times 10^{6}$ cells provides a good prediction of $\bar{N}_{p}$, whereas the coarser grid used for the regular impeller slightly overshoots the value of $\bar{N}_{p}$ with respect to the finer one. On the other hand, the computations based on the dissipation provide an estimate for $\bar{N}_{p}$ that is slightly lower than the impeller power in all cases. This probably stems from the numerical dissipation of the computational method. As expected, the finer grids provide a more accurate energy balance. For the regular impeller, the energy balance is better than the fractal impeller, when the results of the fine grids are compared, despite the higher number of cells used for the fractal impeller. The reason may lie in the flow field differences around the impeller. In contrast to the two large trailing edge vortices observed behind the blades of the regular impeller, a number of smaller vortices emerge in the wake of the ragged edges of the fractal blades. 
Since the energy containing structures are smaller in this case, this may require a finer grid to correctly capture the dissipative scales.

TABLE II. Averaged power numbers for the cases computed at $R e=320$ and the balance between energy injection and dissipation. Reg. stands for regular and Fra. for fractal.

\begin{tabular}{l||c|c|c|c}
\hline \hline Blade type, mesh size & Reg., $4 \times 10^{6}$ & Reg., $13 \times 10^{6}$ & Fra., $9 \times 10^{6}$ & Fra., $21 \times 10^{6}$ \\
\hline Impeller power & 2.36 & 2.32 & 2.31 & 2.32 \\
Dissipation & 2.24 & 2.27 & 2.20 & 2.25 \\
Difference & $5.2 \%$ & $2.2 \%$ & $4.9 \%$ & $3.1 \%$
\end{tabular}

For the simulations at $R e=1600$, the statistical convergence of the power number is shown in Figure 11 and the final values are listed in Table III. Any change in the running averaged values of $\bar{N}_{p}$ in the period after 60 revolutions is less than $\pm 0.37 \%$ for the regular and $\pm 0.48 \%$ for the fractal impeller, with respect to the final values of averaging. Hence, the computations are ended after 100 revolutions and assumed statistically converged.

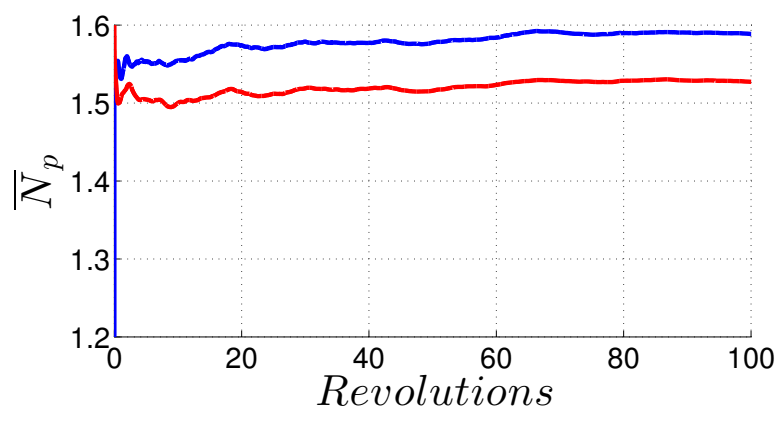

(a) Regular impeller.

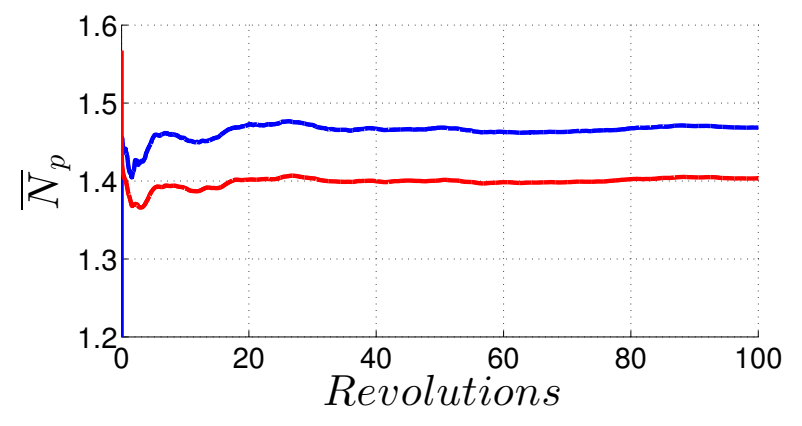

(b) Fractal impeller.

FIG. 11. Running average of the power number over number of revolutions at $R e=1600$, based on impeller torque (blue line) and on dissipation (red line).

Unlike the simulations at the lower $R e$, the results at $R e=1600$ demonstrate a notable difference when the power numbers of the regular and fractal impellers are compared. This difference is comparable to the one observed in the laboratory experiments of Steiros et al. ${ }^{58}$ They measured the power draw using exactly the same two impeller shapes as considered in the present study, in a hexagonal unbaffled tank. They observed $11-12 \%$ lower $\bar{N}_{p}$ for the fractal impeller compared to the regular impeller at $R e=1-2 \times$ $10^{5}$. The numerical estimates at $R e=1600$ suggest a reduction of power consumption of ca. $8 \%$ based on the impeller torque, when the fractal blades are utilized. This 
finding is promising for the efficiency improvement of industrial applications in case the mixing performance of the impeller is also enhanced or remains unchanged. An effort to understand this reduction in power has been made by Steiros et al. ${ }^{58}$ In the remainder of this paper we concentrate in explaining the characteristic frequencies in the power fluctuations.

TABLE III. Averaged power numbers for the cases computed at $R e=1600$ and the balance between energy injection and dissipation.

\begin{tabular}{l||c|c}
\hline \hline Blade type, mesh size & Regular, $60 \times 10^{6}$ & Fractal, $70 \times 10^{6}$ \\
\hline Impeller power & 1.59 & 1.47 \\
Dissipation & 1.53 & 1.41 \\
Difference & $3.8 \%$ & $4.2 \%$
\end{tabular}

Note that the difference between the power draw and the integral dissipation is around $4 \%$ for the cases at $R e=1600$. This corresponds to a slightly larger energy imbalance compared to that achieved at $R e=320$, yet still acceptably small.

Finally, numerically predicted power numbers are compared with the empirical correlation employed to display the variation of $\bar{N}_{p}$ vs. Re in Figure 4. This curve provides the following estimates: $\bar{N}_{p}=2.18$ at $R e=320$ and $\bar{N}_{p}=1.41$ at $R e=1600$, for the geometry of the regular impeller used in this work. These are in qualitative agreement with our results but are lower than our estimates based on the impeller power, by $6 \%$ at $R e=320$ and $12 \%$ at $R e=1600$. The details of the experiments or the uncertainty of the correlation are not provided by Furukawa et al. ${ }^{60}$ However, considering the high number of independent parameters such as the tank and impeller diameters, heights, clearance etc., a degree of discrepancy is inevitable. Moreover, the blade thickness is not considered by this correlation, and is known to have a strong influence on the power number. For example, Rutherford et al. ${ }^{63}$ suggested a correlation between the power number and the blade thickness of a Rushton turbine under fully turbulent regime: $\bar{N}_{p}=6.405-55.673(t / D)$, where $\mathrm{t} / \mathrm{D}$ is the blade thickness to impeller diameter ratio. For instance, if this ratio is reduced from $2 \%$ to $1 \%, \bar{N}_{p}$ would have an increase of ca. $10 \%$ according to this correlation. Although this equation is not directly applicable to our case, it emphasizes the influence of the blade thickness. Taking this into account, we can consider the empirical 
correlation given by Furukawa et al. ${ }^{60}$ only for approximate comparison.

The DNS study conducted by Verzicco et al. $^{30}$, which was already used for code validation purposes, reported ca. 18\% difference between the power input and the integral energy dissipation. The DNS study by Gillissen and Van den Akker ${ }^{31}$ investigated the flow in a baffled tank stirred with a Rushton turbine at $R e=7300$, using the latticeBoltzmann method and $2.9 \times 10^{9}$ grid cells. They reported an energy imbalance of $3 \%$. Considering the high resolution used in the above-mentioned work, having a similar quality in terms of energy balance is regarded as satisfactory. Note that their work employed a uniform Cartesian grid, whereas our simulations utilized a body-fitted unstructured grid which allows a much better cell distribution. This partly explains the smaller mesh sizes in our cases, besides other important factors like the lower Re and lack of baffles in the tank.

\section{ANALYSIS IN THE FREQUENCY DOMAIN}

To the best of authors' knowledge, the fluctuations in the power consumption and their possible fluid mechanical origin have not been discussed in the literature for the present configuration. Labbé et al. ${ }^{64}$ and Bramwell et al. ${ }^{65}$ studied experimentally the fluctuations of power injected into the turbulent flow confined in the cylindrical space between two coaxial counter-rotating disks with eight blades mounted vertically on the surfaces of the disks. Both papers mainly focused on the PDF of the power fluctuations and reported a non-Gaussian distribution skewed towards low values of power. They emphasized the good collapse of PDF of the reduced variable $(P(t)-\bar{P}) / P_{r m s}$ for different Re. Labbé et al. ${ }^{64}$ evaluated the PSD of power fluctuations, where they observed only the peaks related to the impeller rotation frequency and its harmonics, beside a flat region at lower frequencies. They also mentioned that power fluctuations are related to the dynamics of the large scale flow, but did not provide a clear explanation of this relation.

The power fluctuations for the regular impeller are shown in Figure 9 over a short period of time providing a close look at the signal. In Figure 12a, the variation of $N_{p}(t)$ for 80 revolutions is displayed, obtained at $R e=320$ for both regular and fractal impellers. 


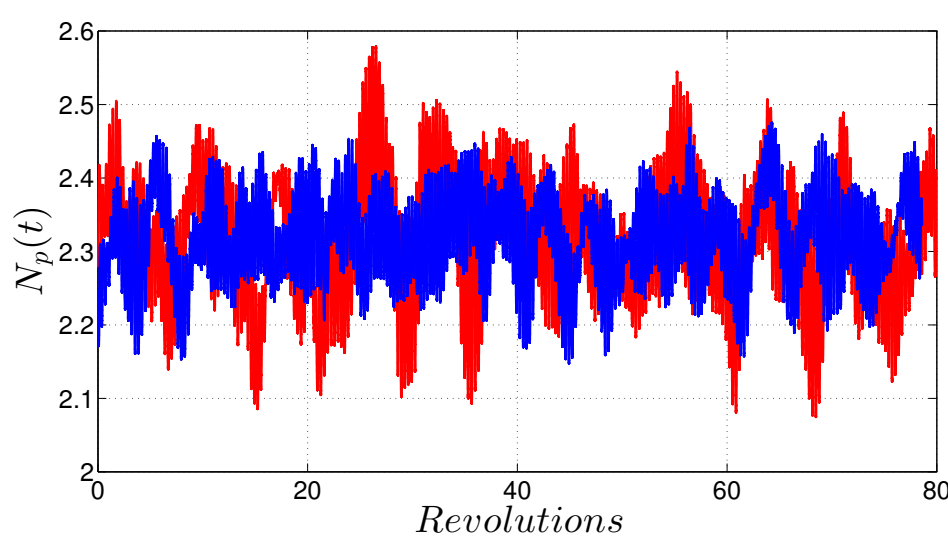

(a) Time series.

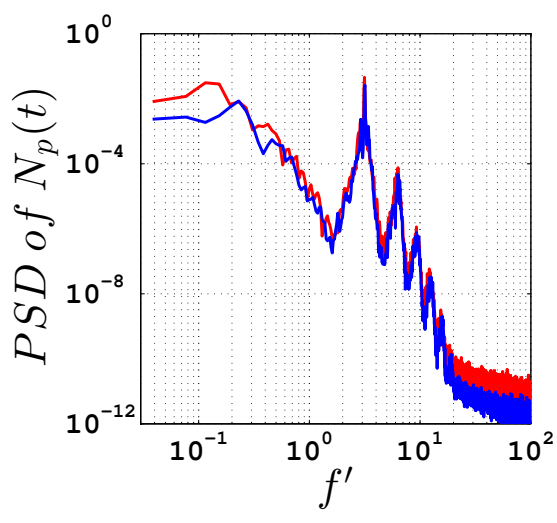

(b) Power spectrum density.

FIG. 12. $N_{p}(t)$ of regular (blue line) and fractal impellers (red line) compared at $R e=320$, over time and frequency domains.

It is observed that the fractal impeller results in higher extrema of $N_{p}(t)$, despite $\bar{N}_{p}$ being equal for both impellers (see Table II). Consequently, the standard deviation of $N_{p}(t)$ normalized by its mean is equal to $3.7 \%$ in the fractal and $2.7 \%$ in the regular impeller case. On the other hand, the power spectra of both cases collapse very well for the frequencies in the range $2<f^{\prime}<20$, where $f^{\prime}$ is a nondimensional frequency, normalized with the impeller rotational speed $\left(f^{\prime}=f / N\right)$. Considering that the signal at the very high frequencies $\left(f^{\prime}>20\right)$ contains very little energy, the main PSD difference appears to be at the very low frequencies. This suggests that the extrema observed in the $N_{p}(t)$ values of the fractal impeller are due to the low frequency fluctuations $\left(f^{\prime}<0.2\right)$.

The PSD of $N_{p}(t)$ of the fractal impeller contains a peak at $f^{\prime} \approx 0.12$ and in the regular impeller case a peak is detected at $f^{\prime} \approx 0.24$ (see Figure $12 b$ ). The low frequency modulation of $N_{p}(t)$ of the regular impeller seen in Figure 9 has a period of ca. four revolutions, possibly related to the aforementioned peak at $f^{\prime} \approx 0.24$. The same peaks are also observed in the PSDs of the torque applied on regular and fractal blades, shown in Figures 19 and 26, respectively. Moreover, the PSDs of axial velocity component acquired in probe points in the discharge region of both regular and fractal impellers show a peak at $f^{\prime} \approx 0.12$ (shown in Figures $15 \mathrm{a}$ and $16 \mathrm{a}$ for the regular impeller case). These low frequency fluctuations may be related to macro-instabilities (MI), discussed at length by many authors ${ }^{66-69}$. Galetti et al. $^{70}$ reported that MIs are observed at $f^{\prime}=0.106$ for 
$R e$ values in the range $400<R e<6300$, based on experiments conducted in a baffled tank stirred with a Rushton turbine with $D / T=1 / 3$. This frequency agrees with the results of further studies concerning pitched-blade turbines ${ }^{71,72}$ and Rushton turbines ${ }^{33,73}$ conducted in the aforementioned range of Re. Although the tank and impeller geometries studied in the present paper are different than the cited papers, the observed low frequency peak in the PSD of axial velocity at $f^{\prime} \approx 0.12$ is close to the MI frequencies reported in the literature. Note that in the regular impeller case the power input has a peak at $f^{\prime} \approx 0.24$, whereas the axial velocity at $f^{\prime} \approx 0.12$, which might be explained as follows: MIs in the upper and lower halves of the tank might be effecting the impeller with exactly the same magnitude because of the symmetry with respect to the mid-height plane, making an impact on the impeller torque with double the frequency of MIs, i.e. $f^{\prime} \approx 0.24$. On the other hand, the fractal impeller does not have the same symmetry property, hence $f^{\prime} \approx 0.12$ is directly reflected in the torque and power fluctuations.

Furthermore, it is also evident in Figure 12b that the strong fluctuations appear at the frequency $f^{\prime} \approx 3$ in both cases regardless of the different blade shapes. Below we attempt to explain the mechanism leading to this peak. To this end, the regular fourbladed impeller at $R e=320$ is considered as the baseline case. After a detailed analysis of this case, the other ones are discussed in order to highlight similarities and differences in the results.

\section{A. Regular four-bladed impeller}

The fluctuations in power are mainly a reflection of the pressure fluctuations applied on the blade surfaces, since it was determined previously that the viscous forces on the blades have a very minor contribution to the impeller torque. These fluctuations are directly linked to the unsteady motion of the trailing vortices. But before their dynamics is analysed, we examine first the shape and position of the time-averaged coherent structures using the mean velocity field.

As mentioned before, one way to illustrate the trailing vortices is to use the $\lambda_{2}$-criterion introduced by Jeong and Hussain ${ }^{11}$. Escudié and Liné ${ }^{10}$ simplified this method so that the 
procedure can be applied on the planar PIV measurements obtained on the radial-axial plane. They ignored the derivatives in the tangential direction as well as derivatives of the tangential velocity component (except $\partial v_{\theta} / \partial \theta$ which was estimated using conservation of mass). This simplification reduces the order of the polynomial equation from third to second, which must be solved in order to calculate the eigenvalues of $\mathbf{S}^{2}+\Omega^{2}$ tensor and decreases the computational cost of this method significantly. Escudié and Liné10 obtained the vortex size and location by means of this simplified approach and found the results to be in very good agreement with the original method introduced by Jeong and Hussain $^{11}$. We have utilized the simplified method to visualise the trailing vortices, as seen in Figure 13. Choosing lower values of $\lambda_{2}$ provides smaller surfaces closer to the vortex cores. Here the isosurfaces of $\lambda_{2} / \Omega^{2}=-0.5$ are selected for a clearer illustration of trailing vortices rather than $\lambda_{2}<0$, where $\Omega$ is the impeller angular velocity.

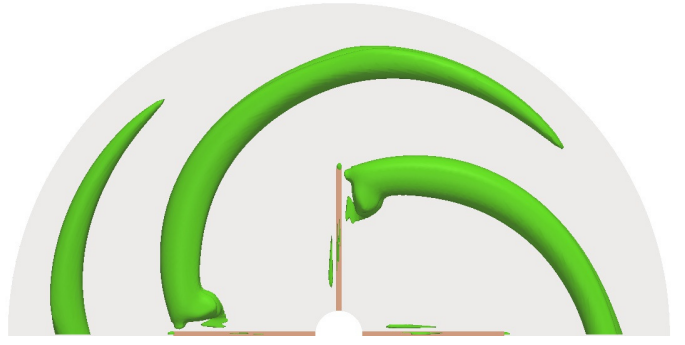

(a) Top view.

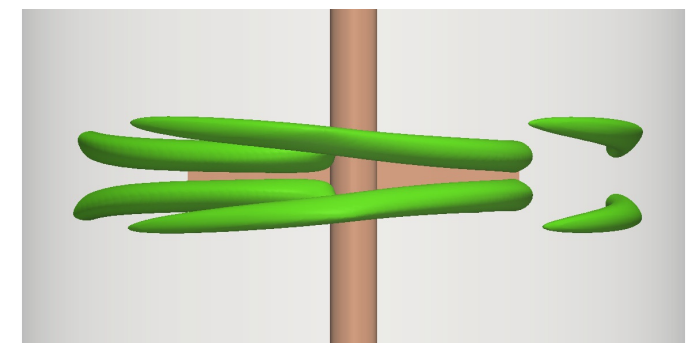

(b) Side view.

FIG. 13. Trailing vortices illustrated by means of simplified $\lambda_{2}$-criterion, $\lambda_{2} / \Omega^{2}=-0.5$.

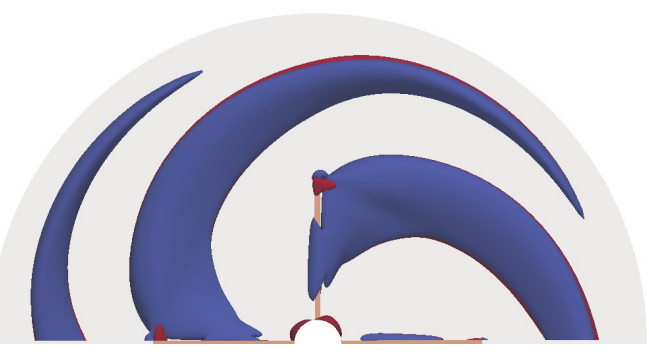

(a) Top view.

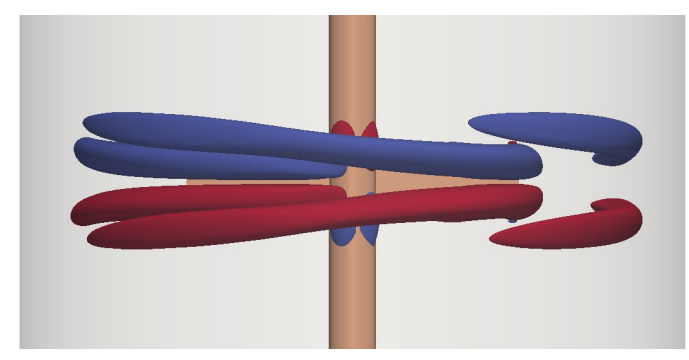

(b) Side view.

FIG. 14. Trailing vortices illustrated with the isosurfaces of normalized tangential vorticity, $\omega_{\theta} / \Omega=1.5$ (red) and $\omega_{\theta} / \Omega=-1.5$ (blue).

In the literature, the mean path of the trailing vortices emerging form a six-bladed Rushton turbine has been illustrated in many studies and a comparison between the results 
of some of them was carried out by Escudié et al. ${ }^{9}$ The structures we observe around the four-bladed impeller are qualitatively in agreement with what was observed by these authors (see Figure 13). Trailing vortices are formed right behind the edges of the blades, initially propagate normal to the blade and then become curved as they are carried with the flow that circulates around the impeller. The side view of the vortices in Figure 13b shows that the vortices are also displaced in the axial direction away from the mid-plane of the tank while being carried in the tangential direction. Since the radial jets are directed upwards or downwards after hitting the tank walls, the mean flow carries the upper and lower branches of trailing vortices along with their motion in the positive or negative axial direction, respectively. Moreover, the time-resolved data shows simultaneous upand-down motion of the vortex pair as well as broken-up parts of isosurfaces impinging on the tank walls.

As an alternative, the trailing vortices are also illustrated by means of the isosurfaces of the tangential component of the vorticity displayed in Figure 14. This has delivered similar structures to those obtained with the simplified $\lambda_{2}$-criterion. In addition to that, the positive or negative values of tangential vorticity indicate the counter-rotation of the vortex pair (positive tangential direction points towards counter-clockwise sense of rotation, for an observer from the top as in Figures 13a and 14a).

In order to understand the relationship between the fluctuations in the power number and the trailing vortices, we recorded the velocity signal at several probe points in the impeller discharge region. It was noticed that the observed peak frequency in the velocity fluctuations can vary with the probe location. Figure 15 shows the PSD of the axial velocity fluctuations and the PSD of the radial velocity fluctuations recorded at probe-1 that lies between a vortex pair at the mid-height plane, hence equidistant from both cores $\left(r=1.27 R, \theta=45^{\circ}\right.$ and $z=0$, as shown in Figure $\left.3 \mathrm{~b}\right)$. The strongest peak found in the PSD of the radial velocity fluctuations at this point is at $f^{\prime}=3.16$ that is exactly the same peak frequency seen in the PSD of the power number fluctuations. On the other hand, the PSD of the axial velocity fluctuations has a peak frequency at $f^{\prime}=1.58$ that is exactly one half of the aforementioned value. Spectra obtained at probe-2 are 
shown in Figure 16. This point is slightly above the first probe so that it is placed in the core of the upper trailing vortex $\left(r=1.27 R, \theta=45^{\circ}\right.$ and $\left.z=0.02 H\right)$. At this point, both axial and radial velocity components oscillate with a dominant frequency of $f^{\prime}=1.58$. Moreover, the magnitude of the PSD of the radial component is almost an order of magnitude higher in the vortex core as seen in Figure 16b. In fact, what we capture at this point is the frequency at which the two cores of the vortex pair grow and shrink in an alternating manner. Similar to the fluctuating lift force of an object due to the vortex shedding in its wake, the axial velocity component fluctuates with the same frequency as the unsteadiness. The radial velocity component at the mid-height is effected by both vortices equally. Therefore this component oscillates with double the instability frequency, like the drag of an object with vortex shedding. Note that the flow field does not contain the periodic fluctuations due to the blade passage that was termed pseudo-turbulence ${ }^{4}$, since the probes have a fixed position with respect to the impeller.

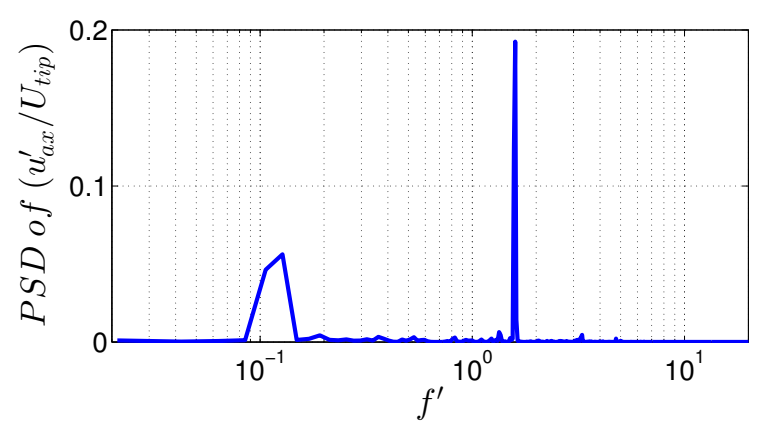

(a) Axial velocity component.

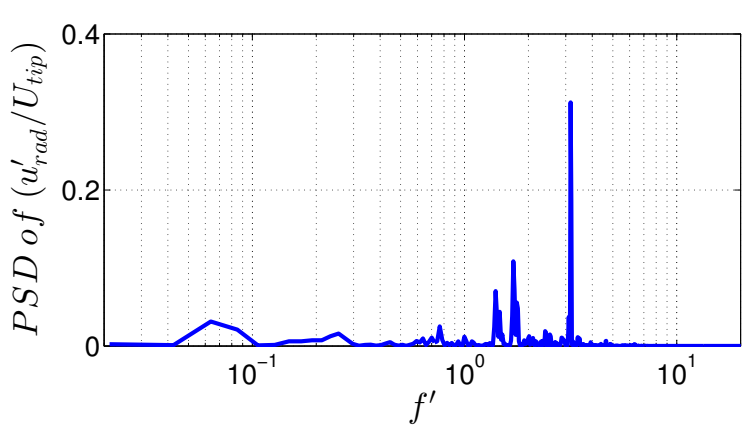

(b) Radial velocity component.

FIG. 15. PSD of velocity fluctuations at the probe- 1 located at $r=1.27 R, \theta=45^{\circ}$ and $z=0$, as shown in Figure $3 \mathrm{~b}$.

The snapshots shown in Figure 17 cover half of the cycle of the unsteadiness at frequency $f^{\prime}=1.58$. In other words, Figures 17a,c,e,g correspond to time instants $t=0, T^{*} / 6, T^{*} / 3, T^{*} / 2$, respectively, with steps of $\Delta t=T^{*} / 6$, with $T^{*}$ being the period of the vortex unsteadiness. Figures $17 \mathrm{a}, \mathrm{c}, \mathrm{e}, \mathrm{g}$ provide information about the generation and evolution of the trailing vortices. The streamlines passing by the upper and lower edges of the blade (shown with blue and red lines, respectively) initially turn towards the suction side of the blade and then bend radially towards the blade tip, due to the 


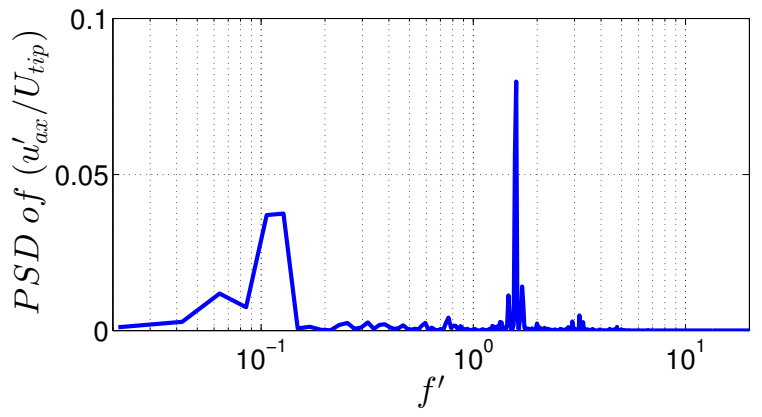

(a) Axial velocity component.

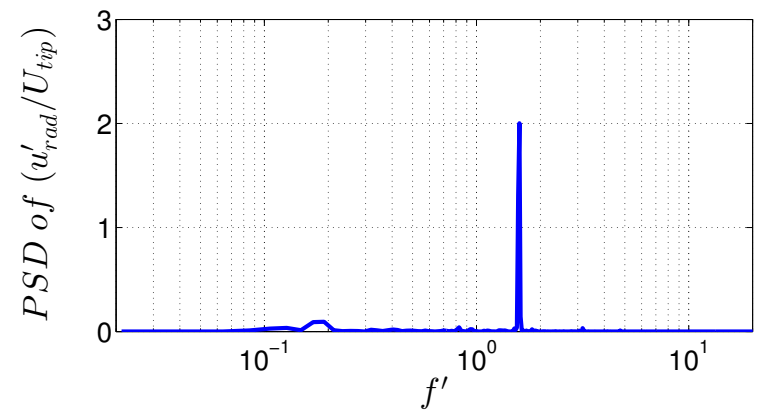

(b) Radial velocity component.

FIG. 16. PSD of velocity fluctuations at the probe-2 located at $r=1.27 R, \theta=45^{\circ}$ and $z=0.02 H$ (slightly above the probe-1, inside the upper vortex core).

radial jet along the suction side of the blade (shown with green lines). Near the blade corners, the blue and red streamlines start rolling around the trailing vortex cores, which the radial jet joins as well, after passing between the vortex pair. We observe that the upper and the lower vortex cores grow and attenuate in an alternating manner (shown with yellow surfaces) while the radial jet swings up and down.

The data analysis also demonstrates that the pressure on the upper or lower half of the suction side decreases while the radial jet is twisted upwards or downwards, respectively. In other words, the radial jet is directed towards the region on the suction side where the pressure is the lowest as illustrated in Figures 17b,d,f,h. This dynamic behaviour of the low pressure region explains the connection between the cycle of vortex unsteadiness and fluctuations in the torque applied on the blade.

So far we have gained some understanding of the phenomena occurring in the wake of one blade. However, the total power consumption is determined by all blades and can also be affected by the phase relation of the instabilities in the wakes of different blades. An instantaneous picture of the coherent structures of two consecutive blades is shown in Figure 18 suggesting that the cycle of unsteadiness with the frequency $f^{\prime}=1.58$ occurs in every blade with a phase shift of $180^{\circ}$ with respect to the preceding (or succeeding) blade. However, the drag force on blades appears with double the frequency $\left(f^{\prime}=3.16\right)$, which results in torque fluctuations at $f^{\prime}=3.16$. These are in the same phase for all blades, which we analyse in the following paragraphs. 


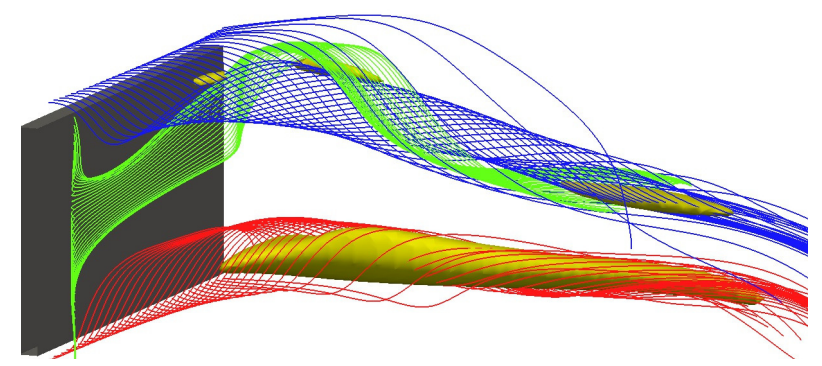

(a) Lower vortex core is at its strongest state.

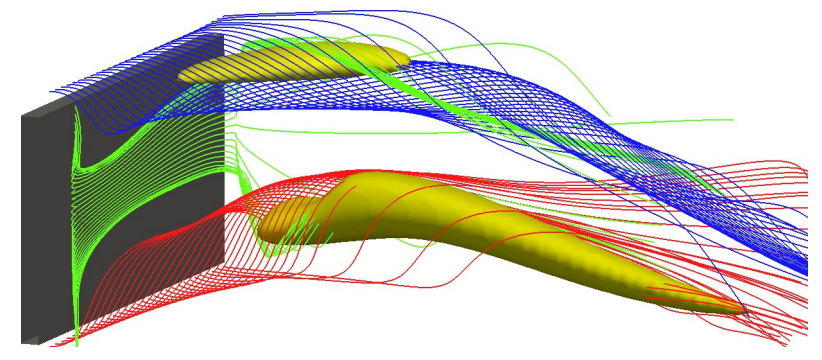

(c) Upper vortex core is growing, as lower core is shrinking.

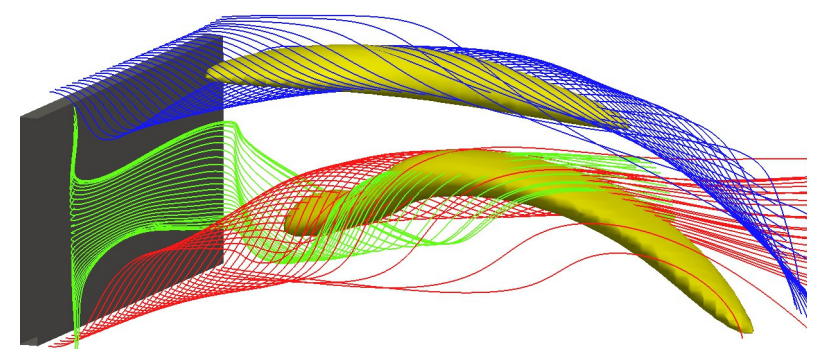

(e) Upper vortex core is growing, as lower core is shrinking.

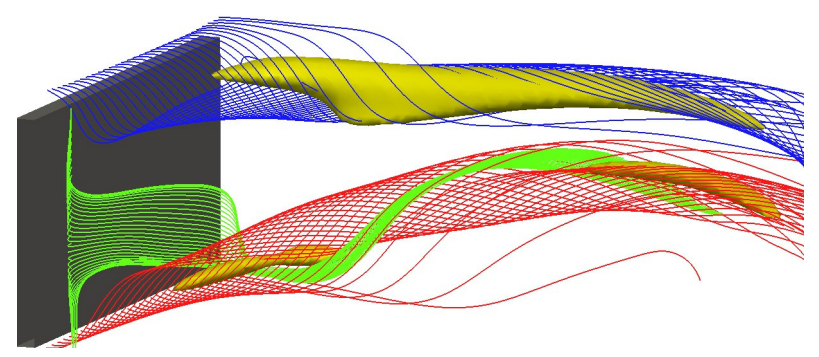

(g) Upper vortex core is at its strongest state.

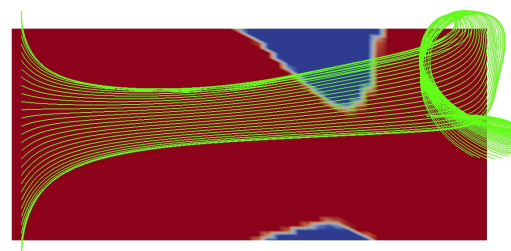

(b) Same instant as (a).

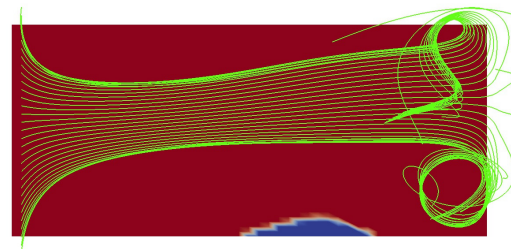

(d) Same instant as (c).

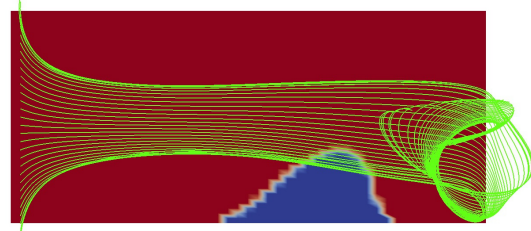

(f) Same instant as (e).

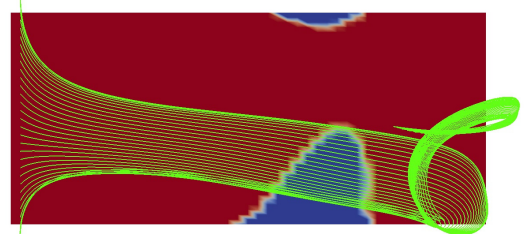

(h) Same instant as (g).

FIG. 17. (a),(c),(e),(g) Isosurfaces of normalized tangential vorticity of $\left|\omega_{\theta} / \Omega\right|=10$ (yellow surfaces) used to illustrate vortex cores. The blue, red and green lines in the images display the streamlines obtained by putting seed points along the upper, lower and inner edges of the blade, respectively. The blade is shown with grey colour. (b),(d),(f),(h) Suction side of the blade is coloured according to the pressure distribution. Red and blue regions illustrate where the pressure is above and below a certain threshold, respectively. 
To evaluate the phase shift between the torque fluctuations on different blades, we recorded the torque caused by each one of the four blades. We refer to the blades with increasing numbers in clockwise direction such that blade 2 encounters the wake of blade 1 etc. Figure 19a displays the PSD of the torque signal of all blades evaluated separately. As expected, all blades yield identical PSD spectra with a peak at $f^{\prime}=3.16$ due to the above discussed phenomenon and another peak at a much lower frequency around $f^{\prime}=0.22$. Nevertheless, the strongest peak of the PSD appears at $f^{\prime}=1.70$ which was not observed in the PSD of the power draw. As seen in Figure 19b, when the torques applied on each one of the four blades are added together, this peak disappears. This suggests the existence of a different instability frequency that is not reflected in the power fluctuations, since it cancels out as the signals from different blades are superimposed.

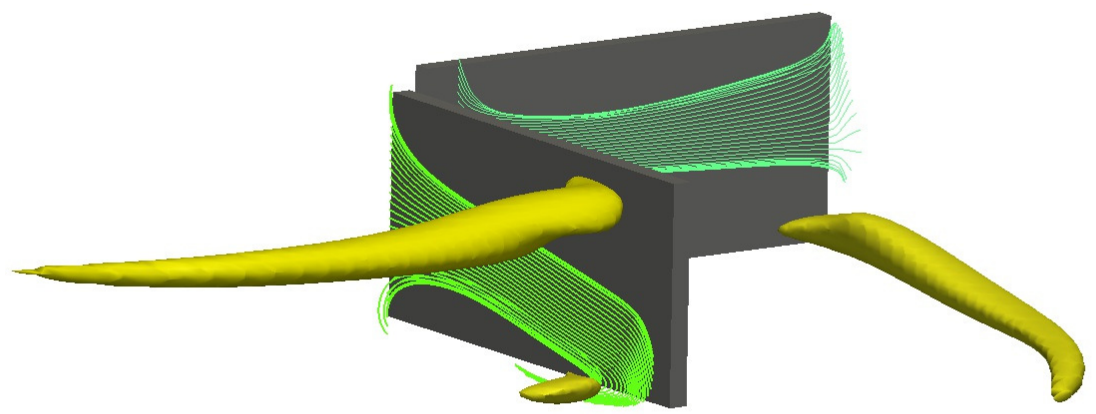

FIG. 18. Streamlines and isosurfaces of normalized tangential vorticity are shown as in Figure 17 for two blades with $90^{\circ}$ angle. Note that the impeller has four blades, but the shaft and the two other blades are removed from the figure for clarity of visualisation.

To further investigate this aspect, the phase relation between the blades 1 and 2 is analysed with the help of Figure 19c. The blue line in Figure 19c shows the PSD of the sum of the torque signals applied on blades 1 and 2. It exhibits the same characteristics of the sum of all blades. On the other hand, the red line represents the PSD of the difference of the torques applied on blades 1 and 2 and demonstrates only the peak at the frequency $f^{\prime}=1.70$. This points at a phase difference of $180^{\circ}$ between the torque fluctuations on blades 1 and 2 at the frequency $f^{\prime}=1.70$ and explains why they cancel each other out.

Furthermore, the phase relation between the blades 1 and 3 is examined in the same way. Figure 19d is the same as Figure 19c but with torque data from blade 3 instead of 
blade 2 . When the torque data from blades 1 and 3 are added, this amplifies the signals at all frequencies. Moreover, they cancel almost completely when subtracted (if the weaker peaks are ignored). This means the signal component at $f^{\prime}=1.70$ on blade 3 is shifted a further $180^{\circ}$ with respect to blade 2 . If the impeller had only the blades 1 and 3 , the unsteadiness at $f^{\prime}=1.70$ would dominate the fluctuations of the power consumption since it has a much higher amplitude than frequency $f^{\prime}=3.16$.

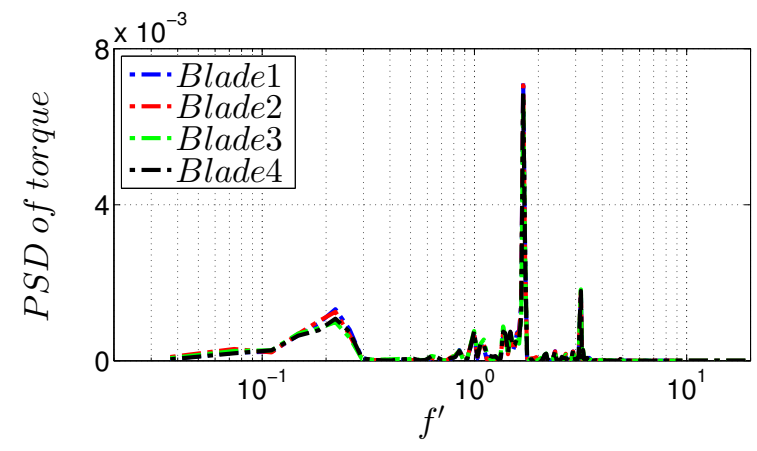

(a) Spectra of four blades.

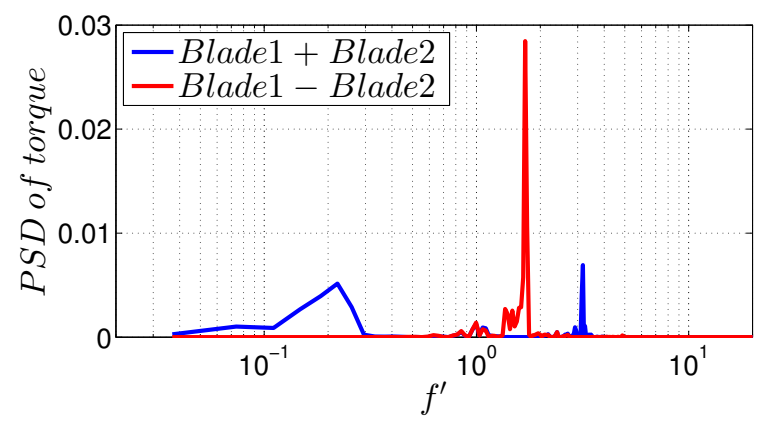

(c) Sum and difference of blades 1 and 2.

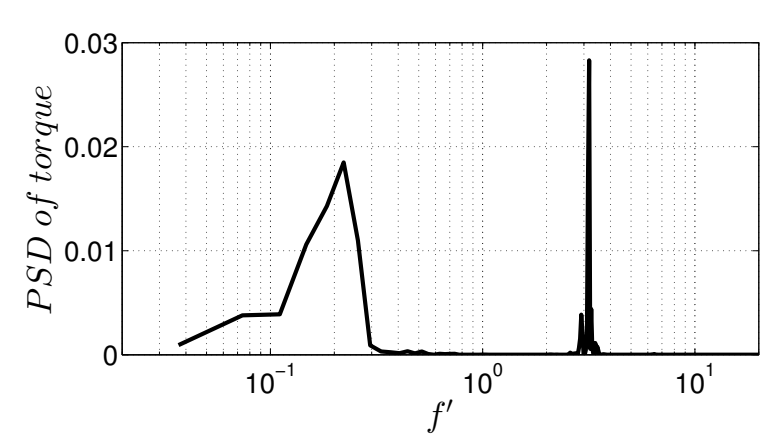

(b) Summation of all blades.

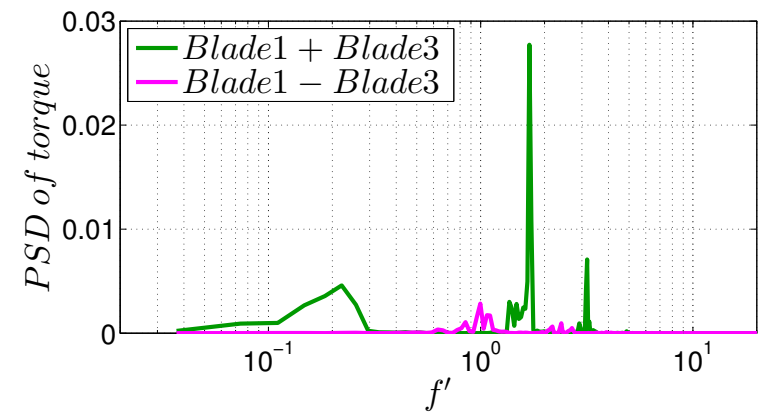

(d) Sum and difference of blades 1 and 3 .

FIG. 19. PSDs of the torque fluctuations applied on the regular blades.

The peak at the frequency $f^{\prime}=1.70$ is also present in the PSD of the radial velocity fluctuations acquired at probe-1 on the mid-height plane, previously shown in Figure 15b. This is the second dominant peak in the PSD after $f^{\prime}=3.16$. However, the other probe point located in the vortex core does not capture any fluctuation at the frequency $f^{\prime}=1.70$. On the other hand, none of the power spectra of the axial velocity fluctuations acquired at these probe points have this peak frequency. These findings imply that there might be a different phenomenon (besides the unsteadiness of the trailing vortex pair), which mainly occurs on the radial-tangential plane around the mid-height leading to an instability at the frequency $f^{\prime}=1.70$. This can be linked to the axial 
vortices in the wake of the blades. Previously, isosurfaces of tangential vorticity were used to illustrate the trailing vortex pair. On the other hand, the isosurfaces of axial vorticity have a quite different shape, as shown in Figure 20. Using the mean velocity field, their time-averaged structures are displayed in Figure 20a indicating an elongated part of the isosurfaces behind the blade tip on the mid-height plane. When the instantaneous structures are observed for several revolutions, it has been detected that this extended part of the isosurfaces grow and detach with the frequency $f^{\prime}=1.70$. Moreover, this occurs in the wake of blades 1 and 3 (or 2 and 4) simultaneously, which agrees with the phase relations of the blades explained above. This can be seen in Figures 20b and 20c. These results suggest that the torque fluctuations at $f^{\prime}=1.70$ can stem from the instabilities of the axial vortices generated on the blade tips. However, the frequency of this mechanism is not reflected in the power draw fluctuations of the four-bladed impeller, which is the main focus this study. Therefore, a more elaborate analysis of this mechanism is not pursued further at this stage.

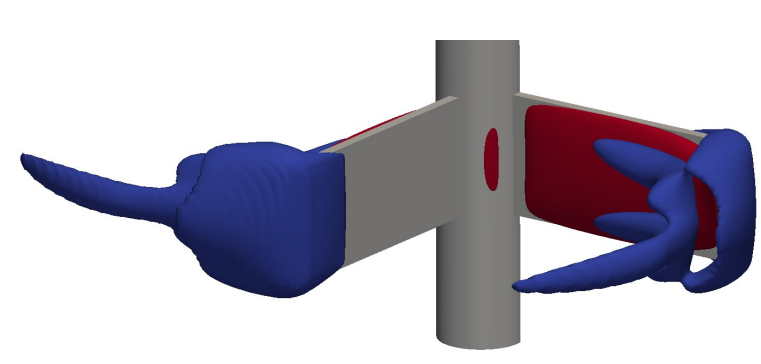

(a)

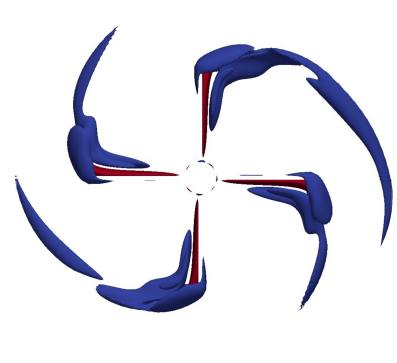

(b)

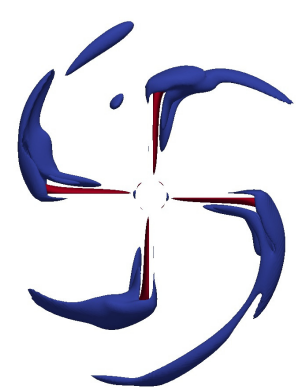

(c)

FIG. 20. Isosurfaces of normalized axial vorticity; $\omega_{z} / \Omega=3.5$ shown with red surfaces and $\omega_{z} / \Omega=-3.5$ with blue surfaces. a) Time-averaged structures, b) and c) instantaneous structures. The impeller is displayed only in the figure on the left side with the grey colour.

\section{B. Regular one-bladed impeller}

In the case of a regular four-bladed impeller, we observed two types of instability mechanisms which are associated with the periodic deformation of the coherent vortical structures. Moreover, we detected a specific phase relation between the wakes of consecutive blades. This may stem from an interaction between the structures emanating from 
different blades. In order to examine whether the generation of instabilities depends on such an interaction, we performed simulations using a regular one-bladed impeller.

The blue curve in Figure 21a displays the power number as a function of the number of revolutions for a one-bladed impeller at $R e=320$. The initial period with large values of $N_{p}(t)$ is a common phenomenon observed with every type of impeller and is due to the starting flow conditions. After this jump, $N_{p}(t)$ decreases and exhibits a plateau. It might have been expected that $N_{p}(t)$ fluctuates in time as observed in the four-bladed case. However, this one-bladed case at $R e=320$ returns a steady laminar flow field around the impeller. Trailing vortex structures emerge again in the wake of the blade but remain static. After approximately 70 revolutions, we artificially superimposed randomly distributed spatial fluctuations to the velocity field. Even though the magnitude of these artificial fluctuations were up to $15 \%$ of the local velocity magnitude, they did not trigger a self-sustaining instability. The absence of any unsteadiness in the one-bladed $R e=320$ case strikingly contrasts the strong fluctuations present in four-bladed $R e=320$ case and implies that the wake interactions have a decisive role in the development of the unsteady behaviour of the coherent structures.

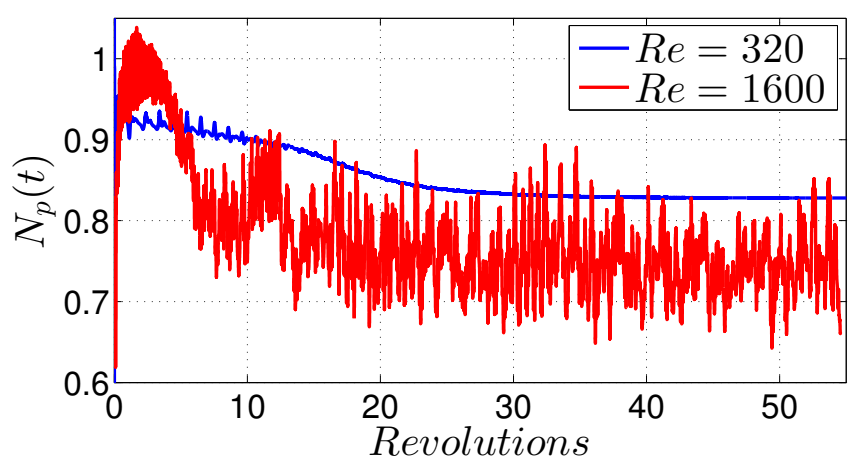

(a)

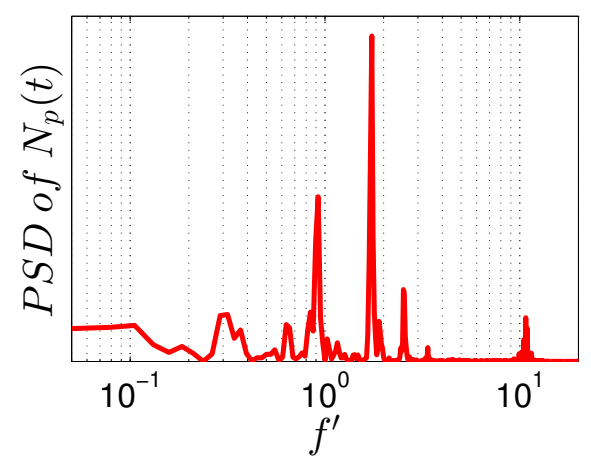

(b)

FIG. 21. $N_{p}(t)$ of the one-bladed impeller plotted against number of revolutions (a), and its PSD for $R e=1600$ (b).

The same simulation was also performed at an increased Reynolds number, i.e. at $R e=$ 1600. As shown by the red curve in Figure 21a, the signal of the power number exhibits strong fluctuations at this Reynolds number. The PSD of power number shows that the dominant peak appears at $f^{\prime}=1.74$ (see Figure 21b). This might be a consequence of 
the same phenomenon at $f^{\prime}=1.70$ linked to axial vorticity which we discussed in the context of the four-bladed impeller in the previous subsection. The slight increase in the frequency (from $f^{\prime}=1.70$ to $f^{\prime}=1.74$ ) can be attributed to the slightly increased relative velocity that the blade tip is exposed to because of the absence of the other blades.

On the other hand, up-and-down swinging motions of the radial jet do not arise in this case, neither the frequency associated with this event (i.e. $f^{\prime}=1.58$ ). Figure 22 illustrates an instantaneous snapshot of the streamlines in the wake of the blade. Note that the radial jet (shown with green lines) is divided into two streams, and these are rolled around the upper and lower vortices. This is unlike the behaviour observed in the four-bladed case where the radial jet swings between upper and lower vortices and rolls around them in an alternating manner, as demonstrated in Figure 17. The lack of this type of unsteadiness cannot be attributed to the increased Reynolds number since this is observed in the case of four-bladed impeller also at $R e=1600$, and is discussed later in the corresponding section. This result supports the conclusion that the interaction between the disturbances generated by multiple blades is essential for the onset of the unsteadiness observed in the four-bladed case.

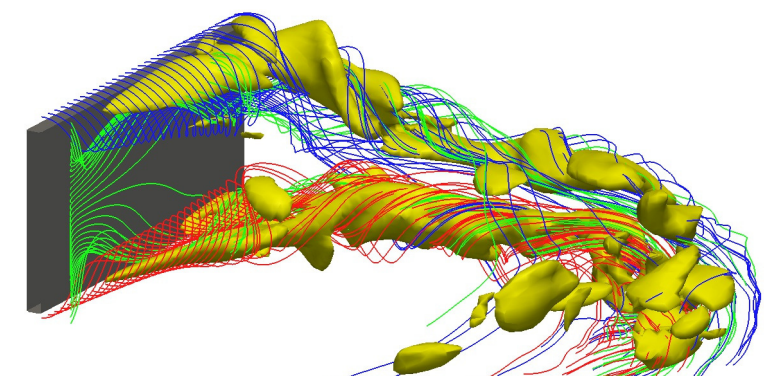

FIG. 22. Yellow surfaces indicate the instantaneous isosurfaces of normalized tangential vorticity of $\left|\omega_{\theta} / \Omega\right|=15$ in the wake of the one-bladed impeller at $R e=1600$. The blade and instantaneous streamlines are as in Figure 17.

\section{Regular two-bladed impeller}

At $R e=320$, the flow around the regular one-bladed impeller is steady without fluctuations. In the tank stirred with a regular two-bladed impeller, the instabilities emerge but grow gradually with a much longer transition time when compared to the 
four-bladed case. Indeed the axial velocity component recorded at probe-1 illustrates this slow growth. As seen in Figure 23, the axial velocity fluctuations kick off after about 60 revolutions and carry on increasing for another 30 revolutions or so.

Consequently, the power number undergoes a similar transition. In the period between 20 and 80 revolutions, $N_{p}(t)$ fluctuates only with the frequency $f^{\prime}=1.74$ with a weak amplitude. However, this amplitude increases gradually during the mentioned period, as shown in Figure 24a. After the axial velocity fluctuations have been amplified after about 90 revolutions, the power number undergoes a transition, as shown in Figure 24b. Shortly after 90 revolutions, additional frequencies are superimposed on the fluctuations of $N_{p}(t)$.

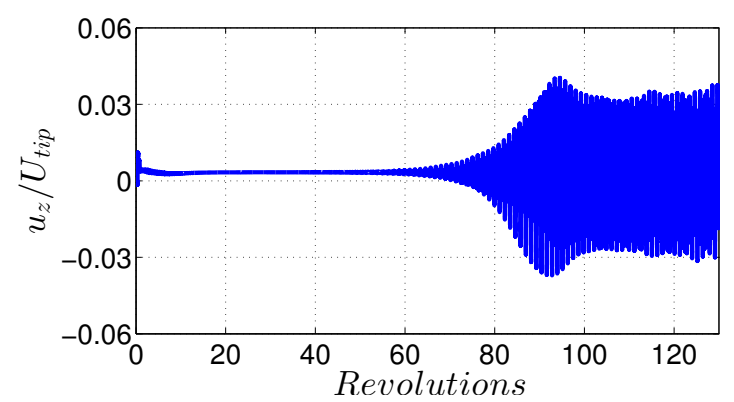

FIG. 23. Instantaneous axial velocity normalized with blade tip speed and plotted against number of impeller revolutions, acquired at probe-1 $\left(r=1.27 R, \theta=45^{\circ}\right.$ and $\left.z=0\right)$.

The trend of the power number is illustrated separately in Figure 25a for the time after the advent of additional frequencies. It is observed that the low frequency modulation of $N_{p}$ starts in an organized periodic manner and slowly becomes rather chaotic as the simulation proceeds, as it is also seen in $N_{p}$ of four-bladed cases. The PSD of the signal, which is obtained using only the part after 130 revolutions, is shown in Figure 25b. The highest peak seen here is at $f^{\prime}=1.74$, the same frequency already detected before the transition. The low frequency fluctuations are at $f^{\prime}=0.18$ and presumably emerge due to the macro-instabilities. The third peak seen in the PSD is at $f^{\prime}=1.58$. We observed that it is caused by the same phenomenon which lead to the fluctuations of $N_{p}(t)$ at $f^{\prime}=3.16$ in the four-bladed impeller case. This was associated with the alternating growth of trailing vortex pair and up-and-down swinging motion of the radial jet. This event occurs in the two-bladed impeller case only with half the frequency (i.e. $f^{\prime}=0.79$ ) when compared to the four-bladed case, which was detected with the help of the PSDs of 
the velocity components acquired in the trailing vortices. This suggests that the number of blades has a direct influence on the frequency of this event. Moreover, it initiates after ca. 100 revolutions simultaneously with the low frequency instabilities, suggesting that this phenomenon might be triggered by the onset of macro-instabilities. On the other hand, the unsteadiness linked to the axial vorticity appears at around $f^{\prime}=1.70$ regardless of the number of impeller blades.

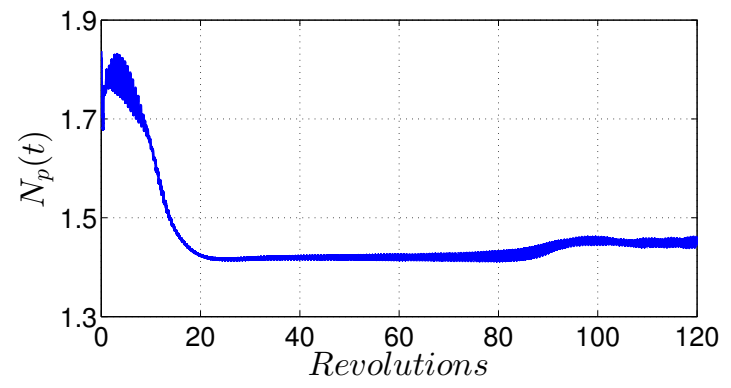

(a)

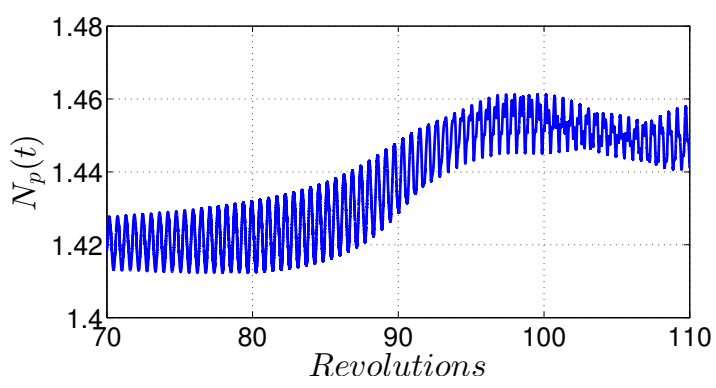

(b)

FIG. 24. $N_{p}(t)$ plotted against number revolutions (a), closer look at the transition (b), for the regular two-bladed impeller case.

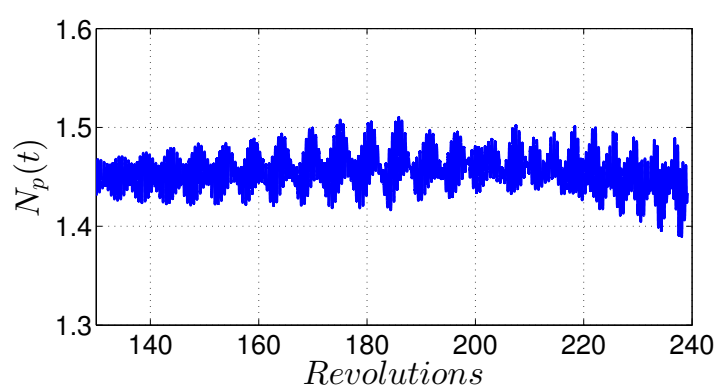

(a)

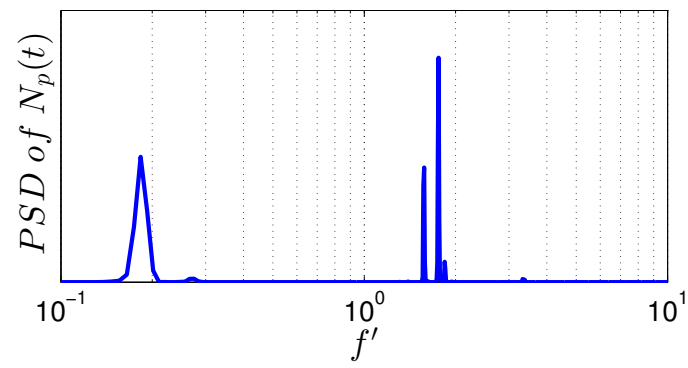

(b)

FIG. 25. $N_{p}(t)$ plotted against number revolutions after the transition (a), PSD of $N_{p}(t)$ using the signal after 130 revolutions (b), for the regular two-bladed impeller case.

\section{Fractal impeller}

At $R e=320$, the cases with the regular and fractal impellers display several common features such as equal power numbers (in time-average sense) and the common peak frequency of power fluctuations, despite different blade geometries. The mechanism leading to the fluctuations in power is already explained in the context of the regular impeller, which is associated with the dynamical behaviour of the trailing vortex pair in the wake 
of the blades. We also observed an unsteadiness linked to the axial vorticity, which has a footprint on the torque signal applied to the individual blades (with $f^{\prime}=1.70$ ) but not on the total power draw, since this signal is cancelled out due to the phase difference between the blades.

When the same analysis was conducted in the context of the fractal impeller, it revealed several differences in the torque applied on the blades with respect to the regular impeller case. The sum of the torque applied on the fractal blades displays fluctuations with a sharp peak frequency at $f^{\prime}=3.14$ and a second peak at $f^{\prime}=0.12$, as indicated in Figure $26 \mathrm{~b}$.

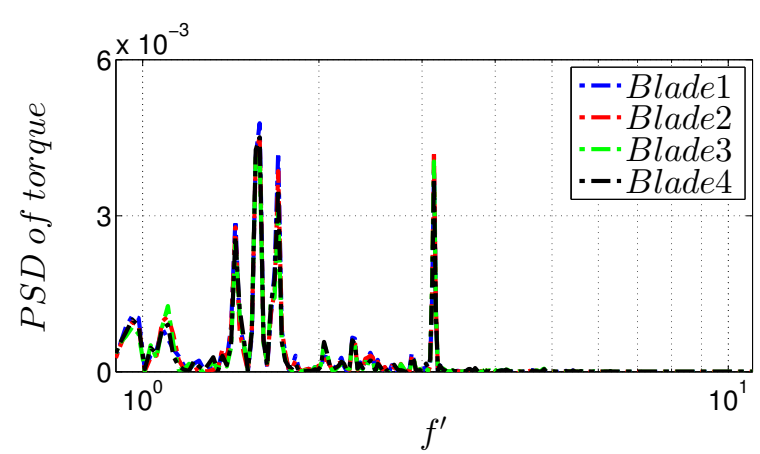

(a) Spectra of four blades.

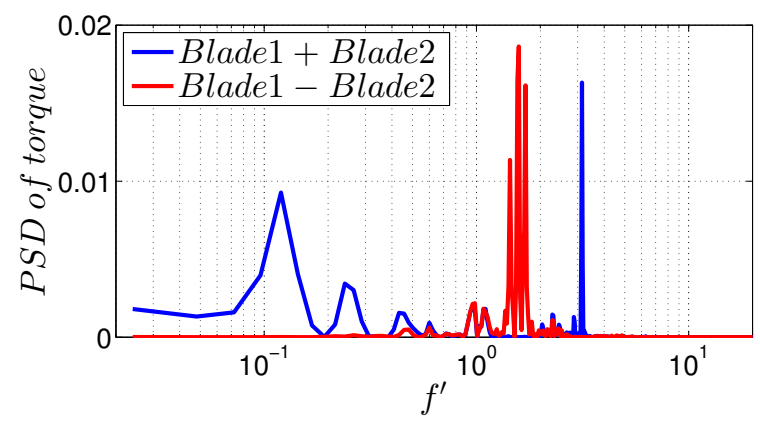

(c) Sum and difference of blades 1 and 2 .

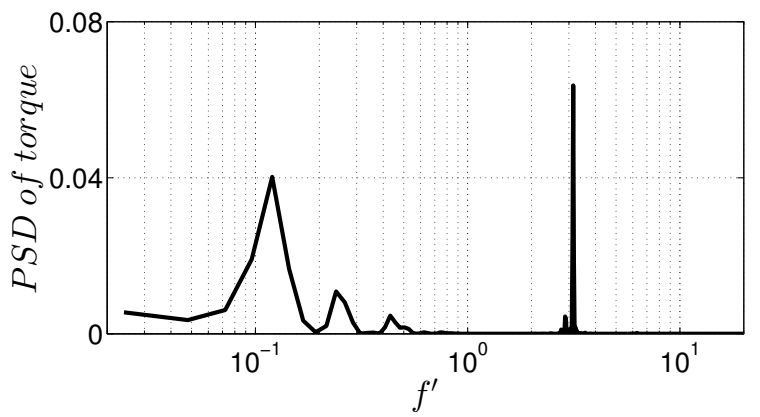

(b) Summation of all blades.

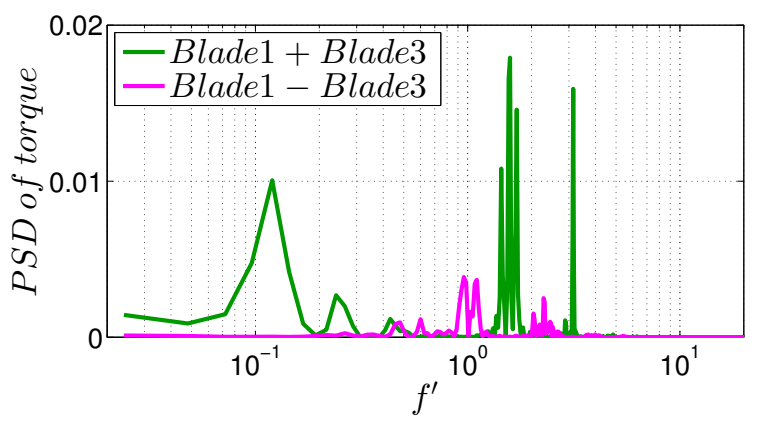

(d) Sum and difference of blades 1 and 3 .

FIG. 26. PSDs of the torque fluctuations applied on the fractal blades.

The high frequency peak in the power draw fluctuations is $f^{\prime}=3.16$ in the regular impeller case, and $f^{\prime}=3.14$ in the fractal impeller case. The difference of $\Delta f^{\prime}=0.02$ is of the order of magnitude of the frequency resolution of the PSD, hence it is negligible. The mechanism leading to this peak frequency is discussed above in the context of regular impeller. This phenomenon was also observed with the same frequency (in multiples of $N$ ) in the fractal impeller case regardless of the fact that the multiple edges of the 
fractal blades generate a higher number of smaller vortices when compared to the regular impeller. This can be seen in Figure 27a where the isosurfaces of tangential vorticity are illustrated using a relatively high value of vorticity which makes it possible to detect the vortex cores. Isosurfaces of lower vorticity levels are shown in Figures $27 \mathrm{~b}$ and $27 \mathrm{c}$. These are suggesting that the multiple trailing vortices tend to interact and merge downstream of the blade.

Figure 26a exhibits the PSD of the torque signal of four fractal blades evaluated separately, focusing on the frequency range $1<f^{\prime}<10$ for clarity of illustration. Besides the peak at $f^{\prime}=3.14$, there are three other peaks with comparable energy levels at $f^{\prime}=1.44, f^{\prime}=1.57$ and $f^{\prime}=1.70$. The peak at $f^{\prime}=1.57$ stems from the same event which gives rise to the peak at $f^{\prime}=3.14$. Unlike regular blades, fractal blades are not symmetrical with respect to the mid-height plane. Consequently, the upper trailing vortex grows larger than the lower one, as seen in Figure 27. Moreover, the upper half of the blade has a larger radial extent than the lower half. Possibly due to these factors the unsteadiness with the frequency $f^{\prime}=1.57$ has a stronger impact on the torque generated on the upper half of the blade compared to the lower half. Therefore the PSD of the torque applied to a blade has peaks at both frequencies $f^{\prime}=1.57$ and $f^{\prime}=3.14$.

Furthermore, we observed that adding the torque applied on two consecutive blades cancels out the signal with $f^{\prime}=1.57$, as shown in Figure $26 \mathrm{c}$ with the blue line (considering that blade 1 and blade 2 are any two blades with $90^{\circ}$ angle between them). This stems from the phase difference between the cycles of both blades. When the low pressure region on the suction side of blade 1 is shifted towards the upper half of the blade, on blade 2 it is shifted towards the lower half. After a half cycle, the described roles of blade 1 and blade 2 are exchanged. When these two instants are compared, the addition of the torque applied on these two blades is equal. In other words, both half cycles apply equally strong torque on the impeller. Therefore, the PSD of the sum of blade 1 and blade 2 presents only the peak at $f^{\prime}=3.14$ and no peak at $f^{\prime}=1.57$.

The other peak at $f^{\prime}=1.70$ was already noted and discussed during the analysis of the regular impeller case. The peak at $f^{\prime}=1.44$ is also associated with the same phenomenon 
as the one at $f^{\prime}=1.70$, namely linked to the instability of the axial vortices emanating from the blade tip. As shown in Figure 28, upper and lower corners of the blade have two separate structures of axial vorticity. Possibly due to different length scales, these vortices lead to fluctuations in torque with two different frequencies. These are in anti-phase when blade 1 and blade 2 are compared and cancel out after the torque applied to these two blades are added, as seen in Figure 26c. However, when any two blades with $180^{\circ}$ are considered, as in Figure 26d, we detected that the signals with frequencies $f^{\prime}=1.44$ and $f^{\prime}=1.70$ are in phase. This phase relation is also observed in the regular impeller case for the unsteadiness caused by the axial vorticity.

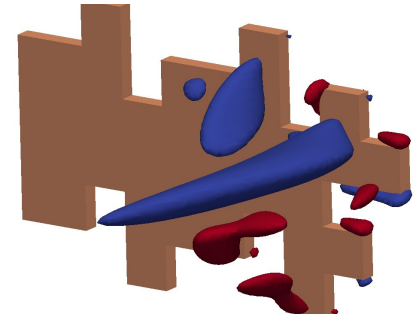

(a) $\omega_{\theta} / \Omega= \pm 7$,

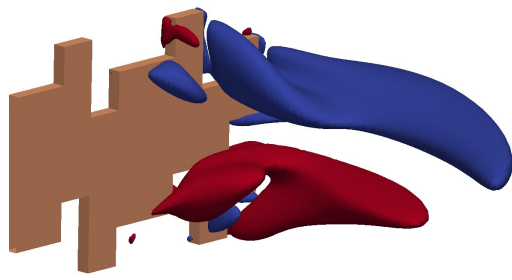

(b) $\omega_{\theta} / \Omega= \pm 5$,

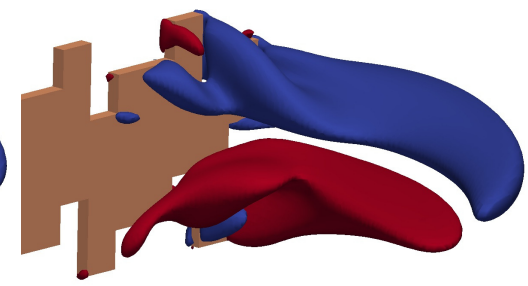

(c) $\omega_{\theta} / \Omega= \pm 4$.

FIG. 27. Isosurfaces of tangential vorticity based on the time-averaged flow field (positive and negative values of vorticity are displayed with red and blue colours, respectively).

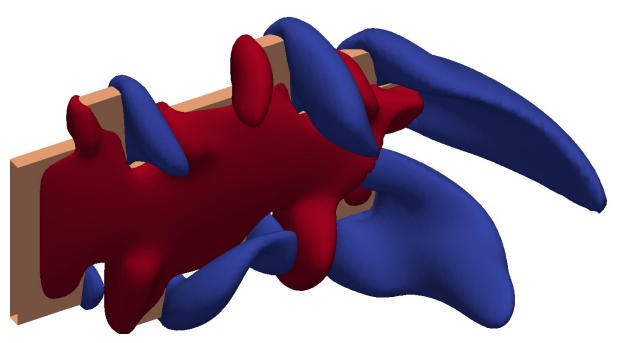

FIG. 28. Isosurfaces of axial vorticity equal to $\omega_{z} / \Omega= \pm 3.5$ based on the time-averaged flow field (positive and negative values of vorticity are displayed with red and blue colours, respectively).

\section{E. Effect of higher Reynolds number on four-bladed impellers}

We already determined that the upper and lower trailing vortices grow in an alternating manner while the radial jet swings up and down at a frequency of $f^{\prime}=1.57-1.58$, for the four-bladed cases at $R e=320$. The results obtained at $R e=1600$ using both 
regular and fractal four-bladed impellers illustrated that the same type of periodic motion of the trailing vortex pair is maintained for the higher Reynolds number. Moreover, this phenomenon occurs at a very similar frequency to what was observed at $R e=320$. To be precise, the frequency is shifted slightly downwards to $f^{\prime}=1.50$ for the cases at $R e=1600$. Steiros et al. ${ }^{58}$ utilized exactly the same two impeller shapes as considered in the present study and conducted time-resolved pressure measurements using pressure transducers fixed on the blade surfaces at $R e=1-2 \times 10^{5}$. They reported that the spectral analysis of the pressure signal has a peak around $f^{\prime}=1.4$ with a high magnitude at blade corners and argued that trailing vortices account for this peak. Considering the decreasing trend of the $f^{\prime}$-value with $R e$, the simulation and experimental results are consistent.

Figures 29a and 29b illustrate the PSDs of the velocity fluctuations in radial and tangential directions, respectively, acquired at probe-2 located inside the upper trailing vortex in the wake of a fractal blade $\left(r=1.27 R, \theta=45^{\circ}\right.$ and $\left.z=0.02 H\right)$. Especially the radial component has a pronounced peak at both Reynolds numbers at the frequencies mentioned above. In both figures the green curve, which represents $R e=320$, has a more prominent peak compared to the blue curve standing for $R e=1600$, with an energy density more than twice as high as the case at $R e=1600$. Although this difference is not apparent due to the log-scaled ordinate of the figure, the log-log plot is preferred in order to illustrate the distribution of energy content over the higher frequencies.

It is clear that at $R e=320$ the magnitude of PSD decreases steeply at higher frequencies and only the large scale structures, namely the trailing vortices are energetic. When the Reynolds number is raised, the energy density at higher frequencies is increased, i.e. the energy of smaller scales. For instance, at $f^{\prime}=15$, which is one order of magnitude greater than the peak frequency, the energy density is ca. four orders of magnitude higher at $R e=1600$ when compared with the case at $R e=320$.

Moreover, the energy spectra of velocity fluctuations follow a slope of $-5 / 3$ in a small extent of frequencies, which was predicted by Kolmogorov ${ }^{74}$ for homogeneous and isotropic turbulence. This was also observed in the context of stirred vessels by other authors: 


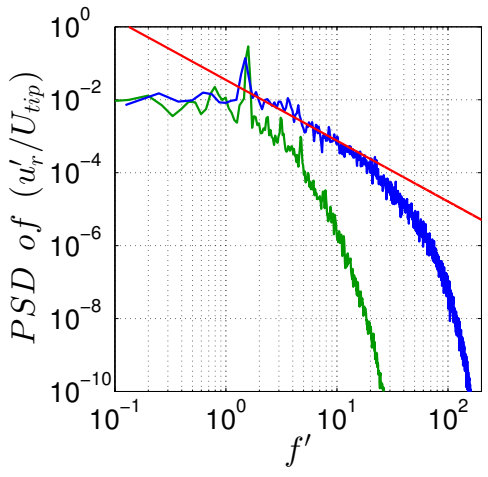

(a) Radial component.

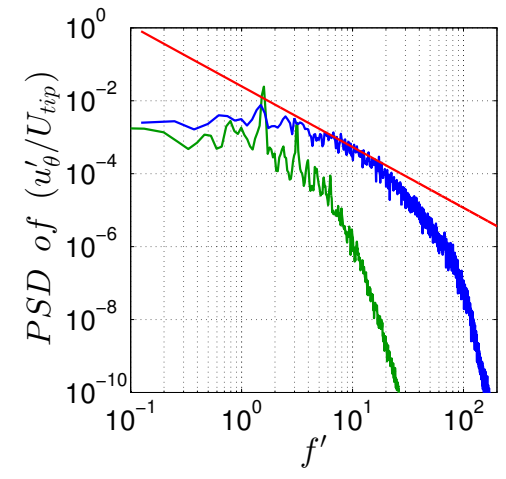

(b) Tangential component.

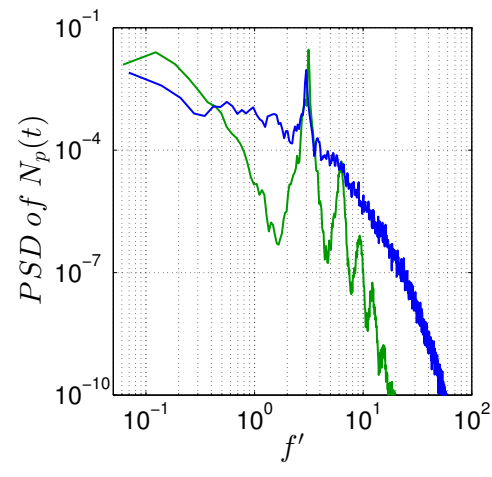

(c) Power draw.

FIG. 29. a) and b) PSDs of velocity fluctuations at probe-2 inside the upper trailing vortex $\left(r=1.27 R, \theta=45^{\circ}\right.$ and $\left.z=0.02 H\right)$, c) PSD of fluctuations of power draw, for the fractal impeller case at $R e=320$ (green line) and $R e=1600$ (blue line). The red line shows the slope of $-5 / 3$ for comparison.

Günkel and Weber ${ }^{75}$ studied the flow in a baffled vessel agitated by a Rushton turbine and captured the turbulent fluctuations in the impeller discharge region for the range of $R e=1-3 \times 10^{4}$. Their work demonstrated energy spectra with a slope of $-5 / 3$ over about 1.5 decades of the frequency space. Lee and Yianneskis ${ }^{7}$ also reported the same observation for a similar configuration at $R e=4 \times 10^{4}$, despite the lack of isotropy and homogeneity in the studied cases. Taking these into account, the presented results imply that the flow in the wake of the blade may become locally turbulent already at $R e=1600$.

If the part of the energy spectrum with a slope of $-5 / 3$ is considered as the cascade of turbulence energy towards smaller scales, this cascade starts around the frequency which is associated with the unsteadiness of trailing vortices. It was already emphasized that the trailing vortices account for the turbulence kinetic energy inside stirred vessels ${ }^{9,12,13}$. However, the process of turbulence production is not completely explained. Our findings provide insights about how the velocity fluctuations are introduced by the trailing vortices and can help to explain the mechanism of turbulence production inside stirred vessels.

Figure 29c compares the PSDs of the power draw fluctuations of the fractal impeller at both Reynolds numbers. It can be noted that the same peak frequency is kept at the increased Reynolds number beside the slight shift from $f^{\prime}=3.14$ to $f^{\prime}=3.0$ which is exactly twice the frequency of the trailing vortex pair unsteadiness for the reasons 
explained earlier for the lower Re case. This demonstrates that the direct connection between the power consumption and the motion of trailing vortices is not broken at the increased Reynolds number while the impeller discharge flow shows indications of local turbulence. All the aspects discussed in this section are common for the regular and fractal impeller cases. This implies that the results are not dependent on the specific blade shape and may be observed even when different types of impellers are employed.

\section{CONCLUSION}

We conducted DNS of the flow inside an unbaffled tank stirred with a radial impeller at $R e=320$ and $R e=1600$. Although we focus on the cases with four-bladed regular and fractal impellers, additional simulations with one- and two-bladed impellers were performed as well, for supporting arguments.

The reliability of the results dealing with four-bladed impellers were verified using the following criteria: (i) A grid convergence study showed that refinement from $9 \times 10^{6}$ to $21 \times 10^{6}$ cells does not affect the quality of the results, for the case of fractal impeller at $R e=320$. (ii) The ratio of cell size to Kolmogorov length scale was kept less than 2 throughout the domain. (iii) The torque applied to the fluid by the impeller was found to be in balance with the torque applied by the tank walls, with an error less than $0.3 \%$ at $R e=320$ and $1.5 \%$ at $R e=1600$. (iv) The imbalance between the energy injection and dissipation was less than $3.1 \%$ at $R e=320$ and $4.2 \%$ at $R e=1600$. Values of $\bar{N}_{p}$ obtained with DNS are somewhat lower than the estimates given by the empirical correlation of Furukawa et al. ${ }^{60}$ (by $6 \%$ at $R e=320$ and $12 \%$ at $R e=1600$ ).

We detected strong fluctuations in the power draw of four-bladed impellers, at the frequencies $f^{\prime}=3.16$ and $f^{\prime}=3.14$, for regular and fractal impellers, respectively, for the cases at $R e=320$. It was determined that these fluctuations are linked to an unsteadiness in the wake of the blades which involves an up-and-down swinging motion of the radial jet while the two cores of the trailing vortex pair grow and decay in an alternating manner. It was also noted that the frequency of the power draw fluctuations is equal to the double of the frequency of the described periodic event. This is because both of the semi-cycles 
of the unsteadiness have a similar impact on the torque applied to the blade. Moreover, the fluctuations in torque applied to the separate blades are in the same phase, hence they are superimposed linearly.

Even though the regular and fractal blades have quite different shapes, the above described unsteadiness occurs with almost the same frequency in both cases, implying that it may appear in cases of other impeller geometries, as well. The frequency of power fluctuations was shifted to $f^{\prime}=3.0$ for both cases at $R e=1600$. Considering that $\bar{N}_{p}$ of the regular impeller decreases from 2.3 to 1.6 when $R e$ increases from $R e=320$ to $R e=1600$, we can conclude that the frequency of power fluctuations is not affected from the change in $R e$ as much as $\bar{N}_{p}$.

It was also observed that the blade number has a strong influence on the above described unsteadiness. The flow field around the one-bladed impeller remained steady at $R e=320$ with a constant power draw over time. Even though it become unsteady at $R e=1600$, the aforementioned phenomenon did not arise. In the two-bladed impeller case at $R e=320$, this motion initiated ca. after 100 revolutions, but this time the unsteadiness frequency was exactly the half of the frequency seen in the four-bladed case. Moreover, its advent occurred simultaneously with the advent of low frequency fluctuations in power draw, possibly due to the so-called macro-instabilities. This implies that macro-instabilities have the potential to trigger an unsteadiness affecting the radial jet and trailing vortices, at a higher frequency than their own.

\section{ACKNOWLEDGEMENTS}

The authors acknowledge the EU support through the FP7 Marie Curie MULTISOLVE project grant no. 317269, the computational resources allocated in ARCHER HPC through the UKTC funded by the EPSRC grant no. EP/L000261/1 as well as the CX2 facility of Imperial College London. 
1 Takashima I, Mochizuki M. Tomographic observations of the flow around agitator impeller. Journal of Chemical Engineering of Japan. 1971;4:66-72.

2 Van't Riet K, Smith JM. The behaviour of gas-liquid mixtures near Rushton turbine blades. Chemical Engineering Science. 1973;28:1031-1037.

3 Van't Riet K, Smith JM. The trailing vortex system produced by Rushton turbine agitators. Chemical Engineering Science. 1975;30:1093-1105.

4 Van't Riet K, Bruijn W, Smith JM. Real and pseudo-turbulence in the discharge stream from a Rushton turbine. Chemical Engineering Science. 1976;31:407-412.

5 Yianneskis M, Popiolek Z, Whitelaw JH. An experimental study of the steady and unsteady flow characteristics of stirred reactors. Journal of Fluid Mechanics. 1987; $175: 537-555$.

6 Stoots CM, Calabrese RV. Mean velocity field relative to a Rushton turbine blade. AIChE Journal. 1995;41:1-11.

7 Lee KC, Yianneskis M. Turbulence properties of the impeller stream of a Rushton turbine. AIChE Journal. 1998;44:13-24.

8 Derksen JJ, Doelman MS, Van den Akker HEA. Three-dimensional LDA measurements in the impeller region of a turbulently stirred tank. Experiments in Fluids. 1999;27:522532.

${ }^{9}$ Escudié R, Bouyer D, Liné A. Characterization of trailing vortices generated by a Rushton turbine. AIChE Journal. 2004;50:75-85.

10 Escudié R, Liné A. A simplified procedure to identify trailing vortices generated by a Rushton turbine. AIChE Journal. 2007;53:523-526.

11 Jeong J, Hussain F. On the identification of a vortex. Journal of Fluid Mechanics. 1995;285:69-94. 
Delafosse A, Morchain J, Guiraud P, Liné A. Trailing vortices generated by a Rushton turbine: Assessment of URANS and large eddy simulations. Chemical Engineering Research and Design. 2009;87:401-411.

13 Derksen JJ, Van den Akker HEA. Large eddy simulations on the flow driven by a Rushton turbine. AIChE Journal. 1999;45:209-221.

14 Bugay S, Escudié R, Liné A. Experimental analysis of hydrodynamics in axially agitated tank. AIChE Journal. 2002;48:463-475.

15 Escudié R, Liné A. Experimental analysis of hydrodynamics in a radially agitated tank. AIChE Journal. 2003;49:585-603.

16 Ducci A, Yianneskis M. Direct determination of energy dissipation in stirred vessels with two-point LDA. AIChE Journal. 2005;51:2133-2149.

17 Escudié R, Liné A. Analysis of turbulence anisotropy in a mixing tank. Chemical Engineering Science. 2006;61:2771-2779.

18 Huchet F, Liné A, Morchain J. Evaluation of local kinetic energy dissipation rate in the impeller stream of a Rushton turbine by time-resolved PIV. Chemical Engineering Research and Design. 2009;87:369-376.

19 Yoon H, Sharp K, Hill D, Adrian R, Balachandar S, Ha M, Kar K. Integrated experimental and computational approach to simulation of flow in a stirred tank. Chemical Engineering Science. 2001;56:6635-6649.

20 Sharp KV, Adrian RJ. PIV Study of small-scale flow structure around a Rushton turbine. AIChE Journal. 2001;47:766-778.

21 Sharp KV, Hill D, Troolin D, Walters G, Lai W. Volumetric three-component velocimetry measurements of the turbulent flow around a Rushton turbine. Experiments in Fluids. 2010;48:167-183. 
Alcamo R, Micale G, Grisafi F, Brucato A, Ciofalo M. Large-eddy simulation of turbulent flow in an unbaffled stirred tank driven by a Rushton turbine. Chemical Engineering Science. 2005;60:2303-2316.

${ }^{23}$ Assirelli M, Bujalski W, Eaglesham A, Nienow AW. Macro- and micromixing studies in an unbaffled vessel agitated by a Rushton turbine. Chemical Engineering Science. 2008;63:35-46.

24 Busciglio A, Caputo G, Scargiali F. Free-surface shape in unbaffled stirred vessels: Experimental study via digital image analysis. Chemical Engineering Science. 2013; 104:868-880.

25 Busciglio A, Grisafi F, Scargiali F, Brucato A. Mixing dynamics in uncovered unbaffled stirred tanks. Chemical Engineering Journal. 2014;254:210-219.

${ }^{26}$ Yang FL, Zhou SJ, Zhang CX, Evans GM, Wang GC. Study of the Turbulent Flow in an Unbaffled Stirred Tank By Detached Eddy Simulation. Chemical Engineering Communications. 2013;200:1347-1365.

27 Scargiali F, Busciglio A, Grisafi F, Brucato A. Mass transfer and hydrodynamic characteristics of unbaffled stirred bio-reactors: Influence of impeller design. Biochemical Engineering Journal. 2014;82:41-47.

28 Yoshida M, Kimura A, Yamagiwa K, Ohkawa A, Tezura S. Movement of Solid Particles on and off Bottom of an Unbaffled Vessel Agitated by Unsteadily Forward-Reverse Rotating Impeller. Journal of Fluid Science and Technology. 2008;3:282-291.

29 Nagata S. Mixing: Principles and applications. New York: Wiley. 1975.

30 Verzicco R, Fatica M, Iaccarino G, Orlandi P. Flow in an impeller-stirred tank using an immersed-boundary method. AIChE Journal. 2004;50:1109-1118.

31 Gillissen JJJ, Van den Akker HEA. Direct numerical simulation of the turbulent flow in a baffled tank driven by a Rushton turbine. AIChE Journal. 2012;58:3878-3890. 
Galletti C, Paglianti A, Yianneskis M. Observations on the significance of instabilities turbulence and intermittent motions on fluid mixing processes in stirred reactors. Chemical Engineering Science. 2005;60:2317-2331.

33 Ducci A, Yianneskis M. Vortex tracking and mixing enhancement in stirred processes. AIChE Journal. 2007;53:305-315.

34 Doulgerakis Z, Yianneskis M, Ducci A. On the interaction of trailing and macroinstability vortices in a stirred vessel-enhanced energy levels and improved mixing potential. Chemical Engineering Research and Design. 2009;87:412-420.

35 Yeoh SL, Papadakis G, Yianneskis M. Numerical simulation of turbulent flow characteristics in a stirred vessel using the LES and RANS approaches with the sliding/deforming mesh methodology. Chemical Engineering Research and Design. 2004; $82: 834-848$

36 Yeoh SL, Papadakis G, Yianneskis M. Determination of mixing time and degree of homogeneity in stirred vessels with large eddy simulation. Chemical Engineering Science. $2005 ; 60: 2293-2302$.

37 Balay S, Abhyankar S, Adams MF, Brown J, Brune P, Buschelman K, Dalcin L, Eijkhout V, Gropp WD, Kaushik D, Knepley MG, McInnes LC, Rupp K, Smith BF, Zampini S, Zhang H. PETSc Users Manual. Tech. Rep. ANL-95/11 - Revision 3.7, Argonne National Laboratory. 2016.

38 Dong L, Johansen ST, Engh TA. Flow induced by an impeller in an unbaffled tank I. Experimental. Chemical Engineering Science. 1994;49:549-560.

39 Wu J, Shu C. Implicit velocity correction-based immersed boundary-lattice Boltzmann method and its applications. Journal of Computational Physics. 2009;228:1963-1979.

40 Gomes-Fernandes R, Ganapathisubramani B, Vassilicos JC. Particle image velocimetry study of fractal-generated turbulence. Journal of Fluid Mechanics. 2012;711:306-336. 
41 Nedić J, Vassilicos JC, Ganapathisubramani B. Axisymmetric turbulent wakes with new nonequilibrium similarity scalings. Physical Review Letters. 2013;111:1-5.

${ }^{42}$ Weitemeyer S, Reinke N, Peinke J, Hölling M. Multi-scale generation of turbulence with fractal grids and an active grid. Fluid Dynamics Research. 2013;45:061407.

43 Nagata K, Sakai Y, Inaba T, Suzuki H, Terashima O, Suzuki H. Turbulence structure and turbulence kinetic energy transport in multiscale/fractal-generated turbulence. Physics of Fluids. 2013;25:065102.

44 Zhou Y, Nagata K, Sakai Y, Suzuki H, Ito Y, Terashima O, Hayase T. Relevance of turbulence behind the single square grid to turbulence generated by regular- and multiscale-grids. Physics of Fluids. 2014;26:075105.

45 Dairay T, Obligado M, Vassilicos JC. Non-equilibrium scaling laws in axisymmetric turbulent wakes. Journal of Fluid Mechanics. 2015;781:166-195.

46 Nedić J, Ganapathisubramani B, Vassilicos JC, Borée J, Brizzi LE, Spohn A. Aeroacoustic Performance of Fractal Spoilers. AIAA Journal. 2012;50:2695-2710.

47 Nedić J, Vassilicos JC. Vortex shedding and aerodynamic performance of an airfoil with multiscale trailing-edge modifications. AIAA Journal. 2015;53:3240-3250.

48 Melina G, Bruce PJK, Vassilicos JC. Vortex shedding effects in grid-generated turbulence. Physical Review Fluids. 2016;1:044402.

49 Nedić J, Supponen O, Ganapathisubramani B, Vassilicos JC. Geometrical influence on vortex shedding in turbulent axisymmetric wakes. Physics of Fluids. 2015;27:035103.

50 Cafiero G, Discetti S, Astarita T. Heat transfer enhancement of impinging jets with fractal-generated turbulence. International Journal of Heat and Mass Transfer. 2014; $75: 173-183$.

51 Suzuki H, Nagata K, Sakai Y, Ukai R. High-Schmidt-number scalar transfer in regular and fractal grid turbulence. Physica Scripta. 2010;T142:014069. 
52 Suzuki H, Nagata K, Sakai Y, Hayase T. Direct numerical simulation of turbulent mixing in regular and fractal grid turbulence. Physica Scripta. 2010;T142:014065.

53 Laizet S, Vassilicos JC. Fractal space-scale unfolding mechanism for energy-efficient turbulent mixing. Physical Review E. 2012;86:1-5.

54 Laizet S, Vassilicos JC. Stirring and scalar transfer by grid-generated turbulence in the presence of a mean scalar gradient. Journal of Fluid Mechanics. 2015;764:52-75.

55 Goh KHH, Geipel P, Hampp F, Lindstedt RP. Flames in fractal grid generated turbulence. Fluid Dynamics Research. 2013;45:061403.

56 Soulopoulos N, Kerl J, Sponfeldner T, Beyrau F, Hardalupas Y, Taylor AMKP, Vassilicos JC. Turbulent premixed flames on fractal-grid-generated turbulence. Fluid Dynamics Research. 2013;45:061404.

57 Goh K, Geipel P, Lindstedt R. Lean premixed opposed jet flames in fractal grid generated multiscale turbulence. Combustion and Flame. 2014;161:2419-2434.

58 Steiros K, Bruce PJK, Buxton ORH, Vassilicos JC. Power consumption and form drag of regular and fractal-shaped turbines in a stirred tank. AIChE Journal. 2017; $63: 843-854$.

59 Nedić J, Ganapathisubramani B, Vassilicos JC. Drag and near wake characteristics of flat plates normal to the flow with fractal edge geometries. Fluid Dynamics Research. 2013;45:061406.

60 Furukawa H, Kato Y, Inoue Y, Kato T, Tada Y, Hashimoto S. Correlation of power consumption for several kinds of mixing impellers. International Journal of Chemical Engineering. 2012;2012.

61 Machado MB, Bittorf KJ, Roussinova VT, Kresta SM. Transition from turbulent to transitional flow in the top half of a stirred tank. Chemical Engineering Science. 2013; 98:218-230. 
${ }^{62}$ Gimbun J, Rielly CD, Nagy ZK, Derksen JJ. Detached eddy simulation on the turbulent flow in a stirred tank. AIChE Journal. 2012;58:3224-3241.

63 Rutherford K, Mahmoudi SMS, Lee KC, Yianneskis M. The influence of Rushton impeller blade and disk thickness on the mixing characteristics of stirred vessels. Chemical Engineering Research and Design. 1996;74:369-378.

${ }^{64}$ Labbé R, Pinton JF, Fauve S. Power fluctuations in turbulent swirling flows. Journal de Physique II. 1996;6:1099-1110.

65 Bramwell ST, Holdsworth PCW, Pinton JF. Universality of rare fluctuations in turbulence and critical phenomena. Nature. 1998;396:552-554.

66 Nikiforaki L, Montante G, Lee KC, Yianneskis M. On the origin, frequency and magnitude of macro-instabilities of the flows in stirred vessels. Chemical Engineering Science. 2003;58:2937-2949.

${ }^{67}$ Roussinova V, Kresta SM, Weetman R. Low frequency macroinstabilities in a stirred tank: scale-up and prediction based on large eddy simulations. Chemical Engineering Science. 2003;58:2297-2311.

68 Hartmann H, Derksen JJ, Van den Akker HEA. Macroinstability uncovered in a Rushton turbine stirred tank by means of LES. AIChE Journal. 2004;50:2383-2393.

69 Doulgerakis Z, Yianneskis M, Ducci A. On the manifestation and nature of macroinstabilities in stirred vessels. AIChE Journal. 2011;57:2941-2954.

${ }^{70}$ Galletti C, Paglianti A, Lee KC, Yianneskis M. Reynolds number and impeller diameter effects on instabilities in stirred vessels. AIChE Journal. 2004;50:2050-2063.

71 Montes JL, Boisson HC, Fort I, Jahoda M. Velocity field macro-instabilities in an axially agitated mixing vessel. Chemical Engineering Journal. 1997;67:139-145.

72 Hasal P, Montes JL, Boisson HC, Fort I. Macro-instabilities of velocity field in stirred vessel: detection and analysis. Chemical Engineering Science. 2000;55:391-401. 
73 Ducci A, Doulgerakis Z, Yianneskis M. Decomposition of flow structures in stirred reactors and implications for mixing enhancement. Industrial and Engineering Chemistry Research. 2008;47:3664-3676.

74 Kolmogorov AN. The local structure of turbulence in incompressible viscous fluid for very large Reynolds numbers. Doklady Akademiia Nauk SSSR. 1941;30:301-305.

75 Günkel AA, Weber ME. Flow phenomena in stirred tanks: Part I. The impeller stream. AIChE Journal. 1975;21:931-939. 